\title{
Bayesian Approach for Analyzing Computer Models using Gaussian Process Model
}

\author{
Hasan Mohammedali Saied ${ }^{1 *}$, Younus Al-Taweel ${ }^{2}$ \\ ${ }^{1 * 2}$ Department of Mathematics, College of Education of Pure Science, University of Mosul, Mosul, IRAQ \\ E-mail: ${ }^{1 *}$ asan.esp81@ student.uomosul.edu.iq, ${ }^{2}$ younus.altaweel@uomosul.edu.iq
}

(Received January 18, 2021; Accepted March 22, 2020; Available online June 01, 2021)

DOI: 10.33899/edusj.2021.129374.1138, () 2021, College of Education for Pure Science, University of Mosul.

This is an open access article under the CC BY 4.0 license (http://creativecommons.org/licenses/by/4.0/).

\begin{abstract}
Mathematical models, usually implemented in computer programs known as computer models, are widely used in all areas of science and technology to represent complex systems in the real world. However, computer models are often so complex in such that they require a long time in computer to be implemented. To solve this problem, a methodology has been developed that is based on building a statistical representation of a computer model, known as a Gaussian process model. As any statistical model, the Gaussian process model is based on some assumptions. Several validation methods have been used for checking the assumptions of the Gaussian process model to obtain the best probabilistic model as an alternative to the computer model. These validation methods are based on a comparison between the output of the computer model and the output of the Gaussian process model for some test data. In this work, we present the Bayesian approach for constructing a Gaussian process model. We also suggeste and compare validation methods that consider the correlation between the output of the computer model and the Gaussian process model predictions with those that do not consider the correlation between these data. We apply the Gaussian process model with the suggested validation methods to real data represented by the robot arm function. We have found that the methods that consider the correlation give more accurate and reliable results. We achieved the calculations using the R program.
\end{abstract}

Keywords: Bayesian Approach, Gaussian Process Model, Computer Model, Cholesky Decomposition, Robot Arm Function.

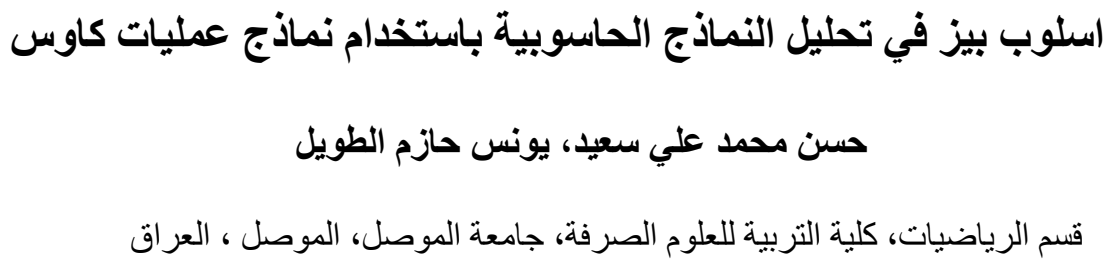


تُستخدم النماذج الرياضية، التي يتم تتفيذها عادةً في برامج الحاسوب المعروفة باسم النماذج الحاسوبية، على نطاق واسع في جميع

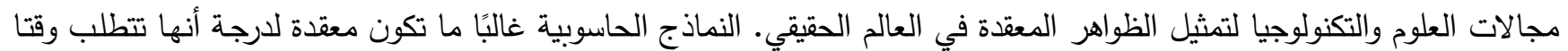
كبيرًا من وقت الحاسوب لتنفيذها. لحل هذه المشكلة، تم تطوير منهجية تعتمد على بناء تمثيل إحصائي للنموذج الحاسوبي، يُعرف باسم نموذج عملية كاوس. نموذج عملية كاوس مبني على بعض الفرضيات، لذا تم استخدام عدة طرق للتحقق من صحة الفرضيات المستخدمة في بنائه من أجل الحصول على أفضل نموذج احتمالي كبديل للنموذج الحاسوبي. تستتد طرق التحقق هذه إلى المقارنة بين مخرجات النموذج الحاسوبي ومخرجات نموذج عملية كاوس لبعض بيانات الاختبار ـ في هذا العمل نقدم اسلوب بيز لبناء نموذج عملية كاوس بالإضافة الى بعض طرق التحقق من صحة الفرضيات التي استخدمت. إضـافة إلى ذللك، نقارن بين طرق التحقق التي تأخذ بنظر الاعتبار الارتباط بين بيانات الاختبار وبين الطرق التي لا تأخذ بنظر الاعتبار الارتباط بين تلك البيانات. تم تطبيق

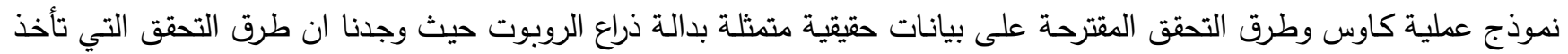
بنظر الاعتبار الارتباط بين بيانات الاختبار تعطي نتائج دقيقة وموثوقة. جميع الحسابات تم اجراها باستخدام برنامج R.

الكلمات المفتاحية: اسلوب بيز، نموذج عملية كاوس، النموذج الحاسوبي، تجزئة كولسكي, دالة ذراع الروبوت.

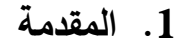
النماذج الحاسوبية (Computer Models) والمعروفة ايضا باسم المحاكيات (Simulators) هي دوال رياضية لنظام رياضي معين أو علاقة معينة يتم تتفيذها في الحاسوب. تم استخدام النماذج الحاسوبية كنماذج بديلة عن الانظمة الواقعية في جميع مجالات العلوم والتكنولوجيا تقريبا [1]. التجارب الواقعية عادة ما تكون مكلفة أو تستغرق وقتا اطول, لذلك فانه يتم عمل محاكاة عن طريق تتفيذ نموذج حاسوبي لقيم مختلفة من البيانات. عادةً ما تكون النماذج الحاسوبية حتمية للإدخال والاخراج, حيث يؤدي تتفيذ النموذج الحاسوبي مرة اخرى لنفس قيم الادخال الى إعطاء المخرجات نفسها دائماً. ان قيم الاخراج غير معلومة من منظور بيز قبل تتفيذ النموذج الحاسوبي لمجموعة ادخال معينة ويمكن التعبير عن عدم اليقين (Uncertainty) حول ناتج النموذج الحاسوبي من خلال عملية عشوائية, اي من خلال تكوين توزيع احتمالي. النتيجة هي تمثيل احصائي لنموذج حاسوبي يعرف بإسم نموذج عملية كاوس ( Gaussian Process (Model [1] و [2] و[3]. وصف]4] كيفية استخدام عملية كاوس لتمثيل دالة غير معروفة, وعمليات كاوس هي الاداة الرئيسية لبناء نموذج عملية كاوس لتمثيل احكامنا حول النموذج الحاسوبي. يمكن بناء نموذج عملية كاوس باستخدام مجموعة من قيم النموذج الحاسوبية تعرف بالبيانات الاولية او بيانات التدريب (Training Data). بمجرد إنشاء نموذج عملية كاوس يمكن إجراء تحليلات مختلفة دون تتفيذ النموذج الحاسوبي مرة أخرى. أبسط هذه التحليلات هو التتبؤ بقيم المخرجات للمدخلات التي لم يتم تتفيذها بالنموذج الحاسوبي. قدم [5] تطبيقا لعمليات كاوس على اختيار المتغيرات والتتبؤ في نماذج حاسوبية. عندما يكون هناك عدم يقين بشأن المدخلات يمكن ان تكون طرق مونتي كارلو (Monte Carlo) المطبقة على النموذج الحاسوبي مكلفة جدا, استخدم [6] نموذج عملية كاوس لتحديد عدم اليقين في مخرجات النموذج الناتجة من عدم اليقين في المدخلات. لاستكشاف كيفية تأثير التغيرات في المدخلات على المخرجات, وصف كل من [7] بعض المقاييس المختلفة لقياس الحساسية باستخدام نموذج حاسوبي. قدم [8] تحليل الحساسية باستخدام نموذج عملية كاوس من منظور استدلال بيز (Sensitivity) 
(Bayesian Inference) نموذج عملية كاوس كبديل للنموذج الحاسوبي, وقدموا ثلاث دراسات حالة حيث تم فيها توضيح تحليل الحساسية وتحليل عدم اليقين. تم استخدام نموذج عمليات كاوس كتقريبات عشوائية لنموذج حاسوبي عالي الكلفة في العديد من مجالات العلوم, لكن بناء نموذج عملية كاوس يتطلب بعض الافتراضات والتقرببات. مالم يكن نموذج عملية كاوس يمثل النموذج الحاسوبي بشكل صحيح, فإن الاستدلالات

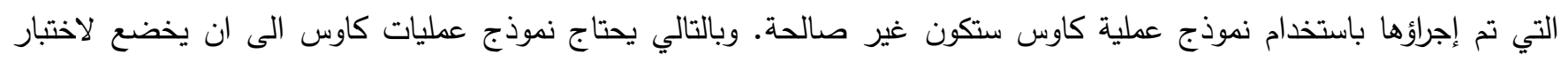
التحقق من صحته (Validation). في هذ البحث نستعرض اسلوب بيز لبناء نموذج عملية كاوس كبديل للنموذج الحاسوبي بالإضافة

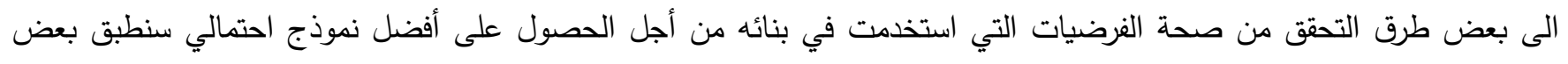
المقاييس العددية والرسومية للتحقق من صحة هذه الفرضيات المستخدمة في بناء نموذج عملية كاوس. كما تم عمل مقارنة بين المقاييس التي تأخذ بعين الاعتبار الارتباط وبين تلك التي لا تراعي الارتباط بين بيانات النموذج الحاسوبي. في القسم الثاني نستعرض الافكار الرئيسية لنموذج عملية كاوس وفي القسم الثالث نصف بإيجاز بعض الإنسان الطرق التي تم اقتراحها

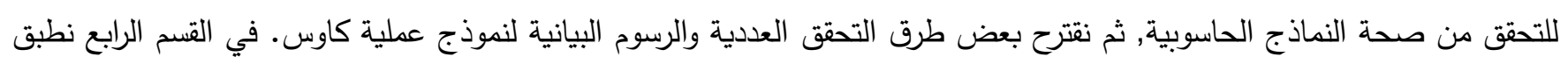

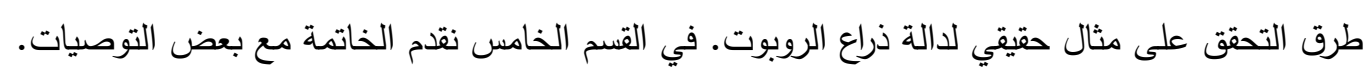
2. نموذج عملية كاوس (Gaussian Process Model):

نموذج عملية كاوس هو عملية عشوائية لتمثيل النموذج الحاسوبي، إذ يُنظر إلى النموذج الحاسوبي على أنه دالة رياضية غير معلومة. على الرغم من أن النموذج الحاسوبي معروف من حيث المبدأ، إلا أن تعقيده يسمح بإعتبار مخرجاته دالة رياضية غير معلومة

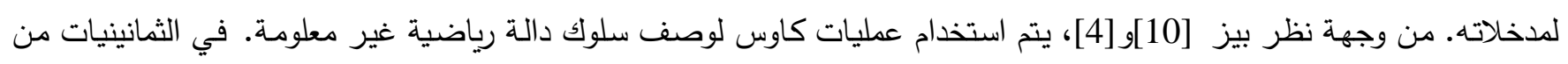
القرن الماضي، تم تقديم الفكرة الأساسية لبناء نموذج إحصائي باستخدام عمليات كاوس في اطار غير بايزي بواسطة [1] وفي اطار بايزي بواسطة [2] و [3].

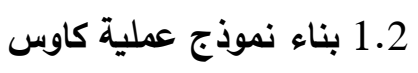
هنا نستعرض بإيجاز الأفكار الرئيسة لبناء نموذج عملية كاوس من منظور بيز , لمزيد من التفاصيل انظر [11] و [12].

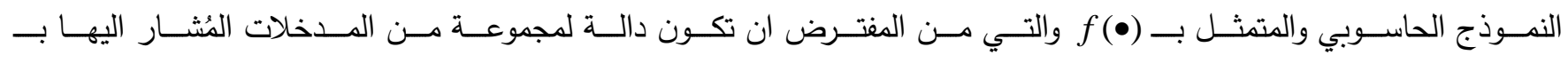

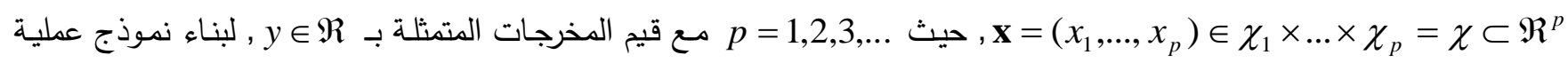

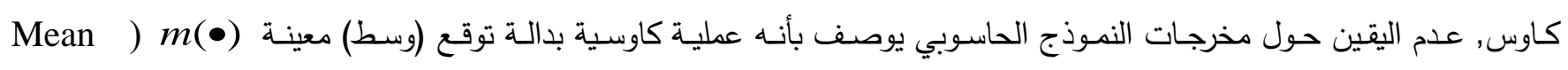

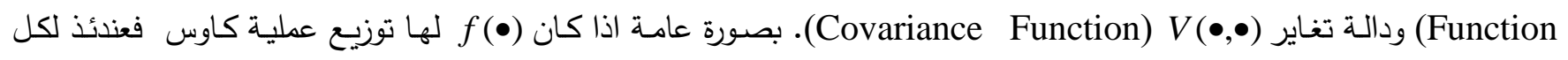
Multivariate Normal ) هو توزيع طبيعي متعدد المتغيرات $n=1,2, \ldots$ (Distribution

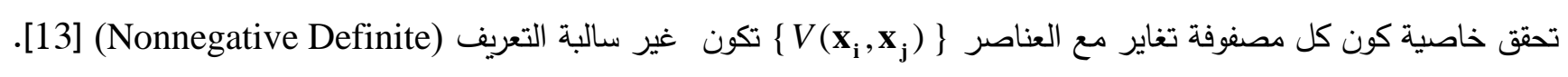
بداية يتم تمثيل التوزيع الاولي حول (•)

$$
f(\bullet) \mid \boldsymbol{\beta}, \sigma^{2}, \psi \sim \mathrm{GP}\left(m_{0}(\bullet), V_{0}(\bullet, \bullet)\right)
$$

$$
m_{0}(x)=h(x)^{T} \boldsymbol{\beta}
$$
حيث ان دالة التوقع (•m 
وان

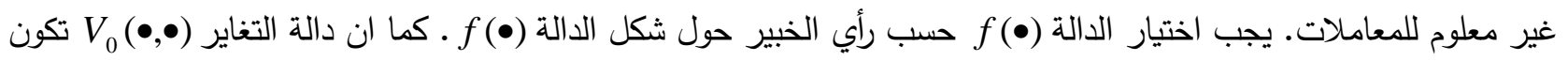

$$
V_{0}\left(x, x^{\prime}\right)=\sigma^{2} C\left(x, x^{\prime} ; \psi\right)
$$

حيث مَ هي معلمة قياس التباين العام غير معلومة و $C(\bullet, \bullet ; \psi$ هي دالة ارتباط معلومة مع معلمة ارتباط غير معلومة. في هذا العمل نستخدم دالة ارتباط كاوس (Gaussian Correlation Function) تسمى معلمات الارتباط. نفرض ان $\left(\psi_{1}, \psi_{2}, \ldots . ., \psi_{p}\right)$ نقاط التصميم لي...... المخرجات توزيع طبيعي متعدد المتغيرات:

$$
\begin{gathered}
\mathbf{y} \mid \boldsymbol{\beta}, \sigma^{2}, \boldsymbol{\psi} \sim \mathrm{N}_{\mathrm{n}}\left(H \boldsymbol{\beta}, \sigma^{2} A\right), \\
H=\left[h\left(\mathbf{x}_{\mathbf{1}}\right), \ldots ., h\left(\mathbf{x}_{\mathbf{n}}\right)\right]^{T}, \\
A_{i, j}=C\left(\mathbf{x}_{\mathbf{i}}, \mathbf{x}_{\mathbf{j}}, \boldsymbol{\psi}\right),
\end{gathered}
$$

$$
\begin{aligned}
f(\bullet) \mid \boldsymbol{\beta}, \sigma^{2}, \boldsymbol{\psi}, \mathbf{y} \sim \mathrm{GP}\left(m_{0}^{*}(\bullet), V_{0}^{*}(\bullet, \bullet)\right), \\
m_{0}^{*}(x)=h(t)^{T} \boldsymbol{\beta}+t(x)^{T} A^{-1}(\mathbf{y}-H \boldsymbol{\beta}) \\
V_{0}^{*}\left(x, x^{\prime}\right)=\sigma^{2}\left[C\left(x, x^{\prime} ; \boldsymbol{\psi}\right)-t(x)^{T} A^{-1} t\left(x^{\prime}\right)\right], \\
t(x)=\left(C\left(x, \mathbf{x}_{1} ; \boldsymbol{\psi}\right), \ldots, C\left(x, \mathbf{x}_{\mathbf{n}} ; \boldsymbol{\psi}\right)\right)^{T} .
\end{aligned}
$$

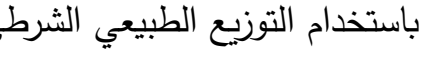

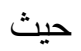

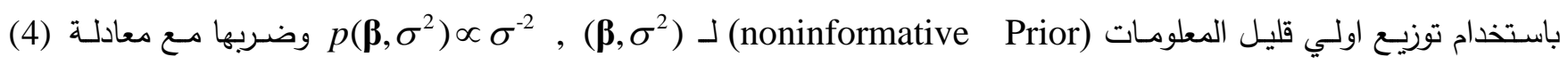

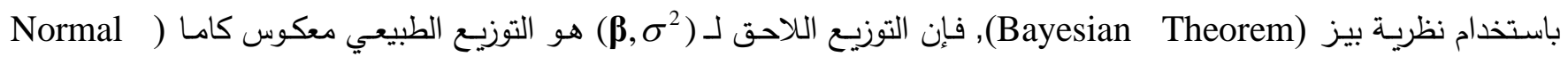
(Inverse-Gamma Distribution

$$
\begin{gathered}
\boldsymbol{\beta} \mid \mathbf{y}, \sigma^{2}, \boldsymbol{\psi} \sim \mathrm{N}\left(\hat{\boldsymbol{\beta}}, \sigma^{2}\left(H^{T} A^{-1} H\right)^{-1}\right), \\
\hat{\boldsymbol{\beta}}=\left(H^{T} A^{-1} H\right)^{-1} H^{T} A^{-1} \mathbf{y}, \\
\sigma^{2} \mid \mathbf{y}, \boldsymbol{\psi} \sim \operatorname{InvGam}\left(\frac{n-q}{2}, \frac{(n-q-2) \hat{\sigma}^{2}}{2}\right),
\end{gathered}
$$




$$
\begin{aligned}
& \hat{\sigma}^{2}=\frac{\mathbf{y}^{\mathbf{T}}\left(A^{-1}-A^{-1} H\left(H^{T} A^{-1} H\right)^{-1} H^{T} A^{-1}\right) \mathbf{y}}{n-q-2} . \\
& \text { بضرب المعادلتين (7) و (8) والتكامل بالنسبة لـ B يمكننا الحصول على (10) } \\
& f(\bullet) \mid \mathbf{y}, \sigma^{2}, \boldsymbol{\psi} \sim \mathrm{GP}\left(m_{1}(\bullet), V_{1}^{*}(\bullet, \bullet)\right) \text {, } \\
& m_{1}(x)=h(x)^{T} \hat{\boldsymbol{\beta}}+t(x)^{T} A^{-1}(\mathbf{y}-\mathrm{H} \hat{\boldsymbol{\beta}}), \\
& V_{1}^{*}\left(x, x^{\prime}\right)=\sigma^{2}\left[C\left(x, x^{\prime} ; \boldsymbol{\psi}\right)-t(x)^{T} A^{-1} t\left(x^{\prime}\right)+\left(h(x)-t(x)^{T} A^{-1} H\right)\right. \\
& \left.\times\left(H^{T} A^{-1} H\right)^{-1} \times\left(h\left(x^{\prime}\right)-t\left(x^{\prime}\right)^{T} A^{-1} H\right)^{T}\right] .
\end{aligned}
$$

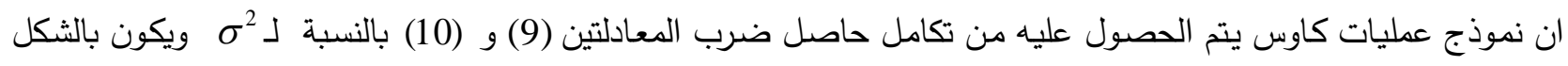

$f(\bullet) \mid \mathbf{y}, \boldsymbol{\psi} \sim \operatorname{Student} \operatorname{process}\left(n-q, m_{1}(\bullet), V_{1}(\bullet, \bullet)\right)$,

$$
V_{1}\left(x, x^{\prime}\right)=\frac{\hat{\sigma}^{2}}{\sigma^{2}} V_{1}^{*}\left(x, x^{\prime}\right) .
$$

تمثل

$$
\begin{aligned}
p(\boldsymbol{\psi} \mid \mathbf{y}) & \propto p(\boldsymbol{\psi}) \iint p\left(\mathbf{y} \mid \boldsymbol{\beta}, \sigma^{2}, \boldsymbol{\psi}\right) p\left(\boldsymbol{\beta}, \sigma^{2}\right) d \boldsymbol{\beta} d \sigma^{2} \\
& \propto p(\boldsymbol{\psi})|A|^{-\frac{1}{2}}\left|H^{T} A^{-1} H\right|^{-\frac{1}{2}}\left(\hat{\sigma}^{2}\right)^{\frac{-(n-q)}{2}}
\end{aligned}
$$

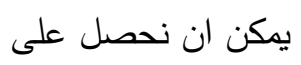

حيث A و

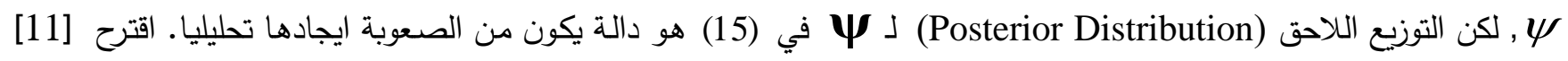

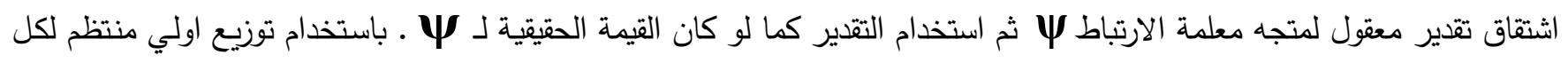
معلمة من معلمات الارتباط فان معادلة (15) تصبح بالثكل التالي

$$
p(\psi \mid \mathbf{y}) \propto|A|^{-\frac{1}{2}}\left|H^{T} A^{-1} H\right|^{-\frac{1}{2}}\left(\hat{\sigma}^{2}\right)^{\frac{-(n-q)}{2}}
$$

وبالتالي فان مقدر الامكان الاعظم (MLE) لمعلمات الارتباط يمكن الحصول عليه بتعظيم المعادلة (16) (16) ـ نموذج عملية كاوس

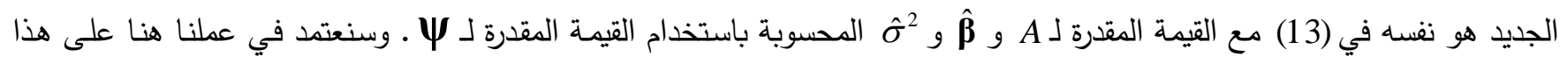

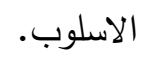

2.2 تصميم توليد البيانات

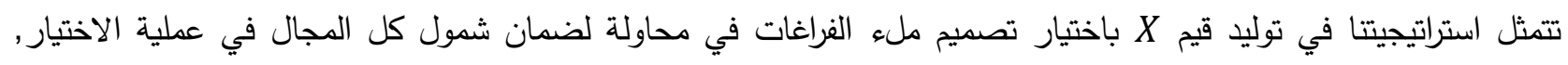

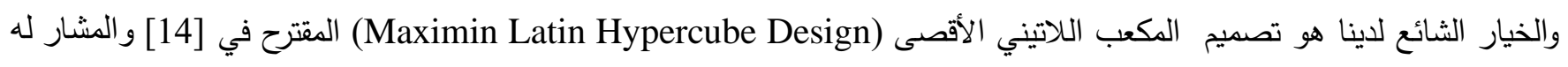


اختصارا Maximin LHD، والذي يتضمن تعظيم الحد الأدنى للمسافة بين أي نقطتي تصميم داخل تصميم المكعب اللاتيني. نغرض

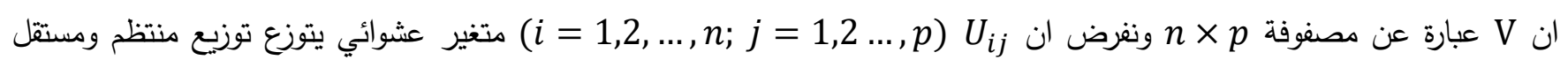

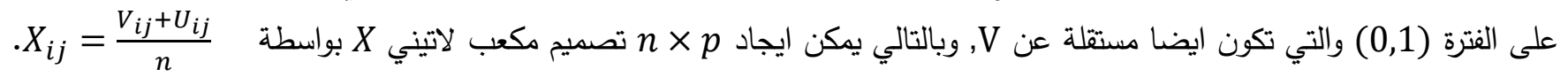
تصميخ Maximin LHD يمكن الحصول عليه من خلال 2.3

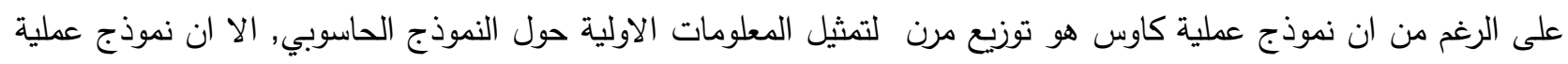

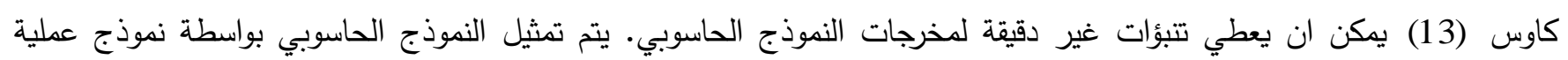

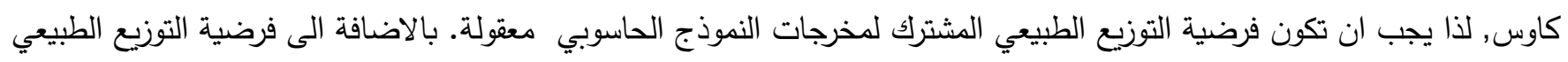

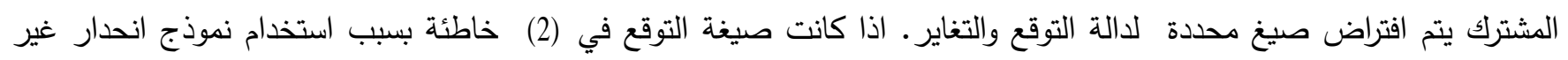

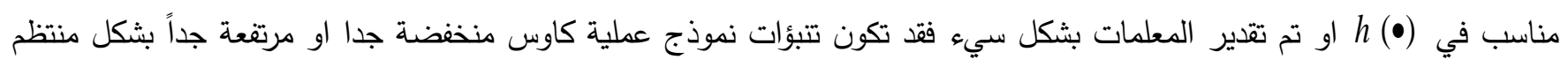

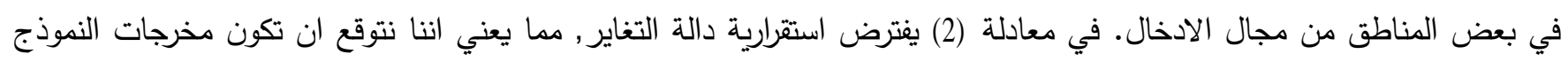

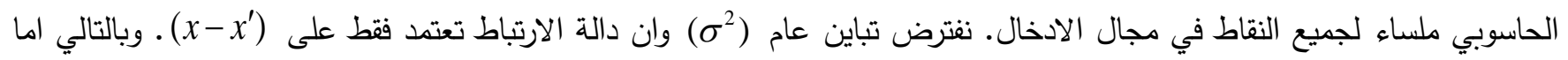

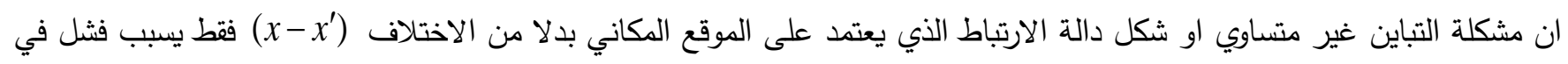

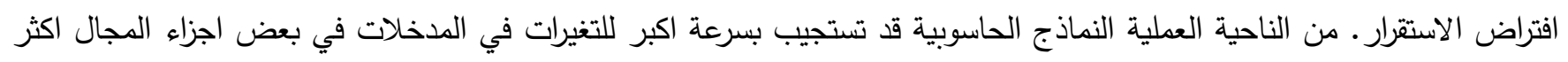

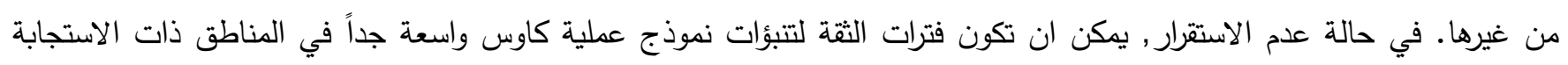

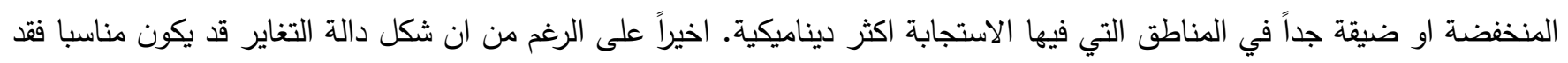
يكون تقدير المعلمات

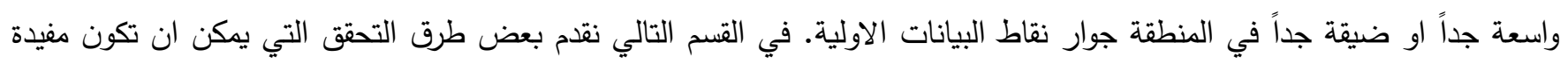

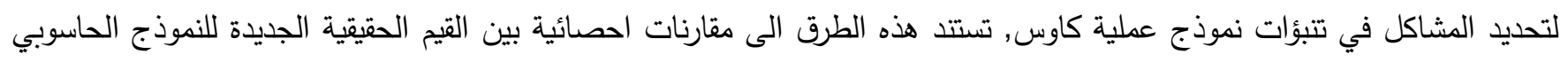

والقيم المتنبئة.

3. طرق التحقق من نموذج عمليات كاوس

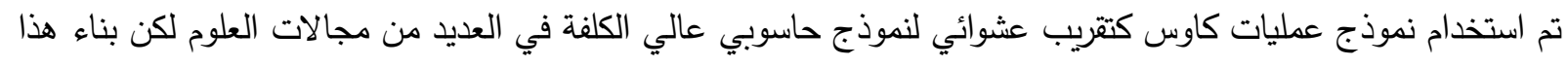

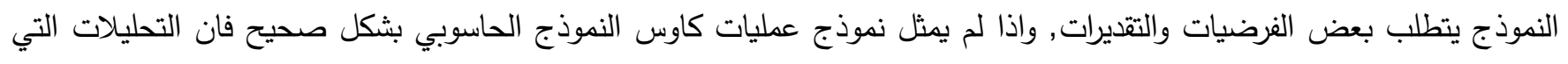

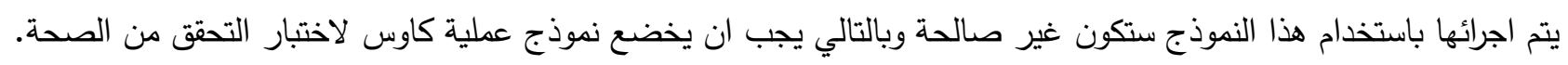

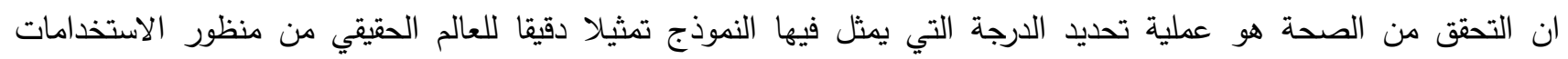

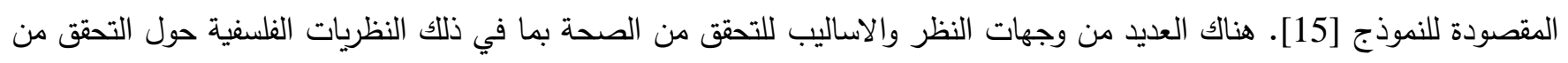

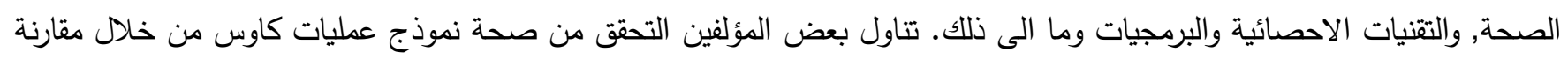

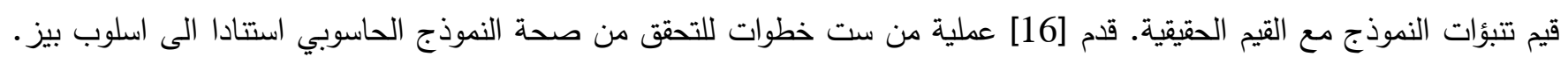

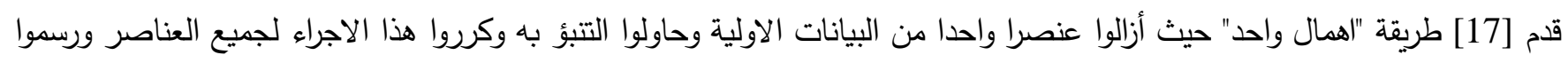

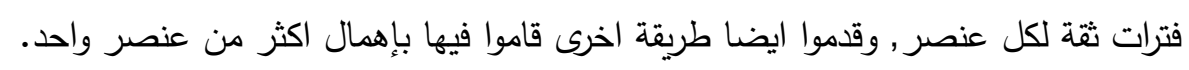
استخدم [11] المخططات البيانية المسماة رسومات Quantile-Quantile Plots) Q-Q للبواقي المعيارية لنموذج المعايرة

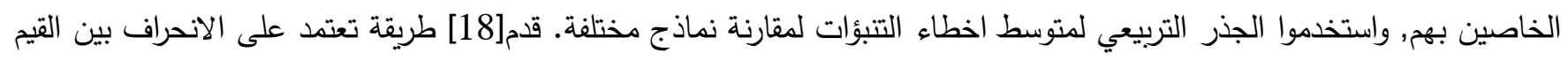




\section{Journal of Education and Science (ISSN 1812-125X), Vol: 30, No: 2, 2021 (148-164)}

الحقيقية وتتبؤات بيز الخطية للنموذج الحاسوبي. تهتم طرق التحقق من الصحة بمقارنة النموذج الحاسوبي بالواقع وتفترض وجود اخطاء مستقلة في البيانات, في حين نركز في العمل الحالي على طرق التحقق من صحة نموذج عملية كاوس كبديل للنموذج الحاسوبي عالي الكلفة وبالتالي فان عملية التحقق الخاصة بنا تستند الى مقارنة نموذج عملية كاوس مع النموذج الحاسوبي. ان تتبؤات نموذج عملية كاوس ليست مستقلة ومن المهم ان تاخذ هذه الطرق الارتباط في نظر الاعتبار .

3.1

يمكن التحقق من صحة نموذج عملية كاوس بدراسة النماذج الخطية ذات الاخطاء غير المستقلة, لان عملية كاوس هي حتمية للنموذج الخطي العام. نظرا لان نموذج عملية كاوس يصوغ دالة حتمية فان التتبؤات بالقيم الحقيقية المستخدمة لبناء النموذج تكون مثالية وبالتالي يمكن الحصول على البواقي باستخدام مجموعة بيانات جديدة, لذلك يجب تكييف طرق التحقق المستخدمة في النماذج الخطية العامة لتكون قابلة للتطبيق في اطار نموذج حاسوبي. في سياق النماذج الخطية العامة قدم [19] نظرية عامة للبواقي واصفا القيم المتبقية الهامشية والثرطية. القيم المتبقية الهامشية(Marginal Residuals) هي الاخطاء بين القيم الحقيقية والقيم المتتبئة في حين ان القيم المتبقية الثرطية(Conditional Residuals) هي الاخطاء بين القيم التتبؤية للقيم الحقيقية الجديدة غير المستخدمة لبناء النموذج. قدم [20] طرق تحقق رسومية لقيم البواقي الهامشية والثرطية في سياق انحدار السلاسل الزمنية, حيث قاموا بتحليل وتجزئة مصفوفة التباين المقدرة للبقايا باستخدام طريقة تجزئة كوليسكي للحصول على بواقي غير مرتبطة بتباين الوحدة. استخدم [21] تحلل

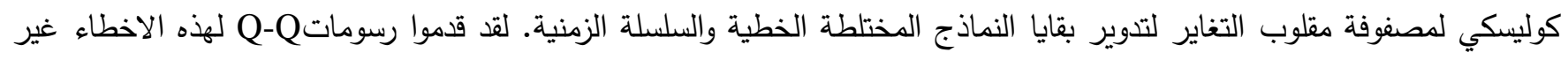
المترابطة مما يوفر خصائص مقاربة للتوزيع التراكمي واخطاء معيارية نقطية. ان نموذج عملية كاوس هو دالة حتمية مما يشير الى عدم وجود اخطاء هامشية كما حددها [19]. على وجه التحديد فان تتبؤات نموذج عملية كاوس للمخرجات المستخدم لبناء النموذج ستساوي تماما المخرجات المعنية مع عدم وجود تباينات. يمكننا الحصول على اخطاء التتبؤ فقط لتنفيذ النموذج الحاسوبي للقيم غير المستخدمة في بناء نموذج عملية كاوس, الاخطاء الثرطية لـ[19]. ميزة اخرى مهمة يجب اخذها في الاعتبار في طرق التحقق من صحة النماذج هي ارتباط الخطأ بسبب بنية الارتباط. في هذا البحث نستخدم طرق التحقق الرسومية التي قدمهاء[20] والذين رسموا الاخطاء الشرطية الغير مرتبطة مقابل ترتيب البيانات ومن قبلهم [21] الذين استخدموا رسم Q-Q للاخطاء الثرطية غير المرتبطة. لقد قام [20] بفهرسة

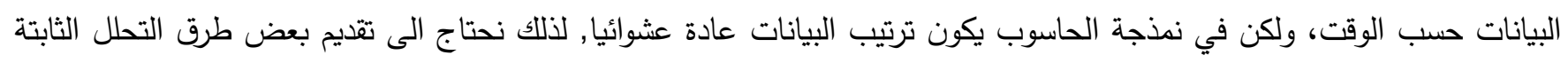
لترتيب البيانات. تحلل كوليسكي (Cholesky Decomposition) يعتمد على ترتيب البيانات وفي هذا العمل نستخدم تحلل كوليسكي

المحوري لبناء الاخطاء غير المرتبطة.

للتحقق من صحة نموذج عملية كاوس تستند طريقتا الى مقارنات بين تتبؤات النموذج والقيم الحقيقية للنموذج الحاسوبي لمجموعة بيانات جديدة. لتكن

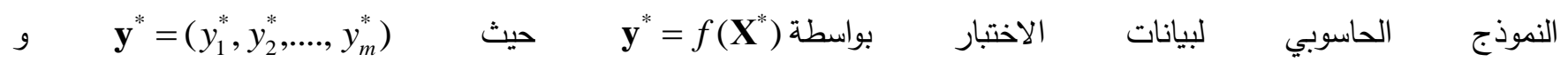
يجب اختيار بيانات الاختبار لتغطي كل مجال الادخال التي نرغب في استخدام نموذج عملية كاوس عليها, خلاف ذلك قد يكون التحقق من صحة نموذج عملية كاوس لمجموعة جزئية معينة فقط من مجال الادخال. ان طريقة التحقق العام (•D) هي دالة لمخرجات بيانات الاختبار وتقترح مقارنة بالبيانات الاولية. D(y

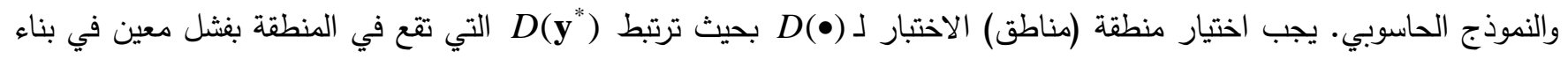


نموذج عملية كاوس. إذا لم تكن هناك مؤشرات على وجود تعارض عبر مجموعة من طرق التحقق هذه فيكننا اعتبار ان نموذج عملية كاوس يمثل النموذج الحاسوبي بدقه.

(خطاء التنبؤ الفردية المعيارية (Individual Standardized Prediction Errors) يتم ايجاد اخطاء التتبؤ الفردية المعيارية لبيانات الاختبار والمخرجات المتتئة لنفس المدخلات مقسوما على جذر التباين:

$$
D_{i}^{I}\left(\mathbf{y}^{*}\right)=\frac{y_{i}^{*}-E\left[f\left(\mathbf{x}_{i}^{*}\right) \mid \mathbf{y}\right]}{\sqrt{V\left(f\left(\mathbf{x}_{i}^{*}\right) \mid \mathbf{y}\right)}},
$$

حيث

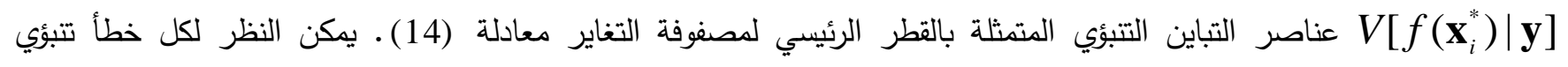

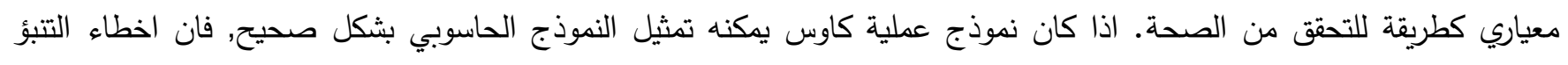

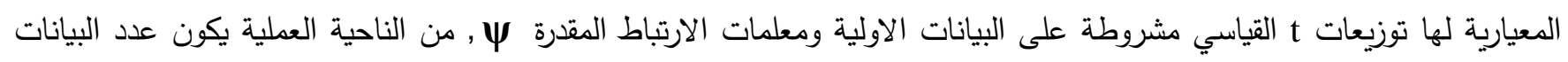

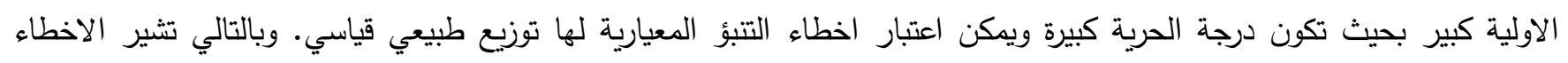

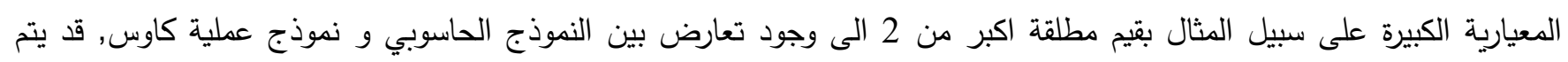

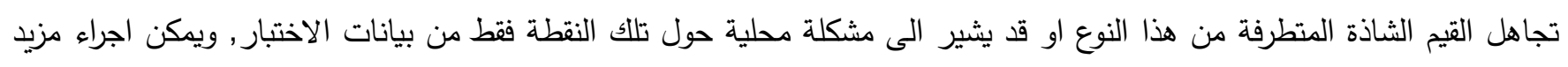

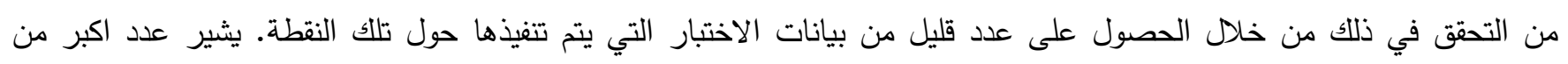

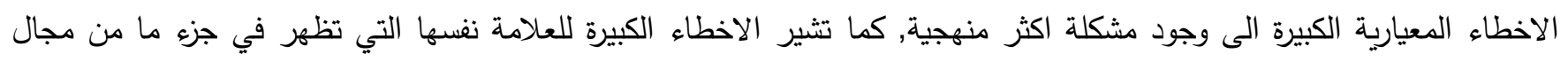

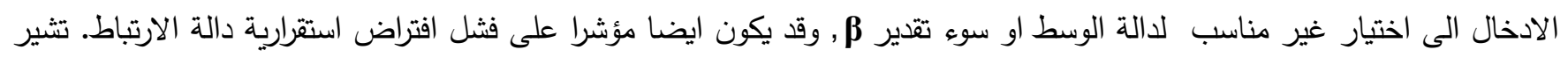

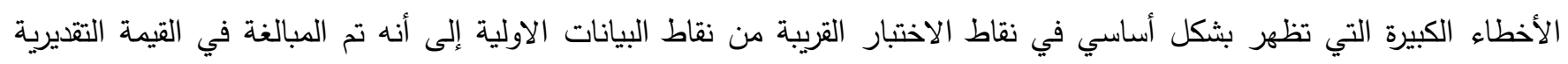

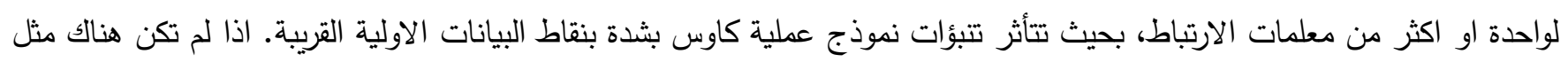

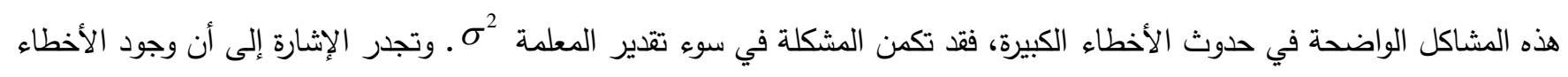

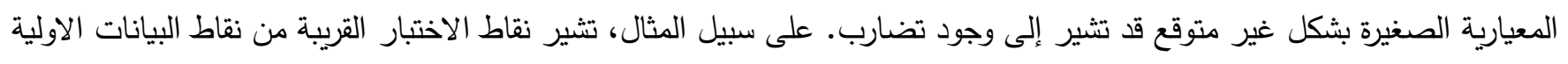

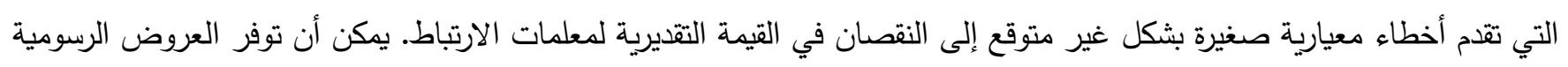

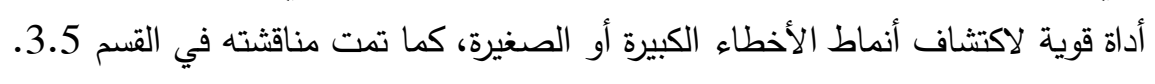

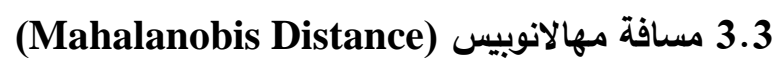

على الرغم من ان مجموعة الاخطاء المعيارية الفردية

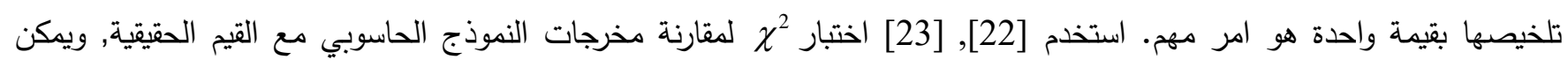
استخدام نفس الفكرة كطريقة تحقق لمقارنة تتبؤات نموذج عملية كاوس بمخرجات النموذج الحاسوبي ضمن نفا لفس المدخلات, طريقة مربع كاي للتحقق من الصحة تعطى بواسطة:

$$
D_{\chi^{2}}\left(\mathbf{y}^{*}\right)=\sum_{i=1}^{m} D_{i}^{I}\left(\mathbf{y}^{*}\right)^{2}
$$

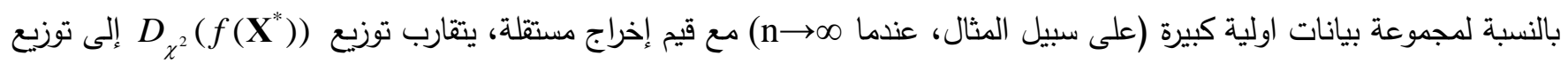

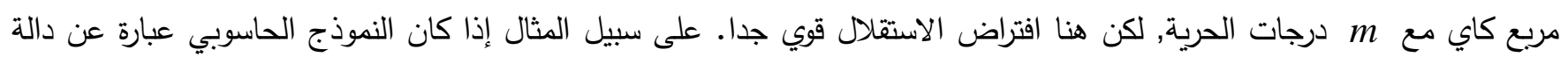


ملساء فمن المتوقع أن تكون المخرجات مماثلة عندما تكون العناصر قريبة من بعضها البعض في مجال الإدخال. يتم التحكم بالارتباط

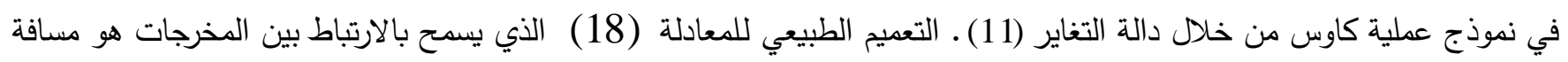

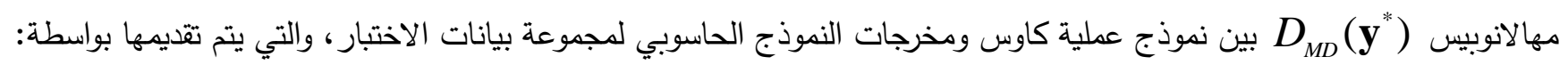

$$
\begin{aligned}
D_{M D}\left(\mathbf{y}^{*}\right)= & \left(\mathbf{y}^{*}-E\left[f\left(\mathbf{X}^{*}\right) \mid \mathbf{y}\right]\right)^{T} \\
& \times\left(V\left[f\left(\mathbf{X}^{*}\right) \mid \mathbf{y}\right]\right)^{-1}\left(\mathbf{y}^{*}-E\left[f\left(\mathbf{X}^{*}\right) \mid \mathbf{y}\right]\right)
\end{aligned}
$$

حيث ان [ل

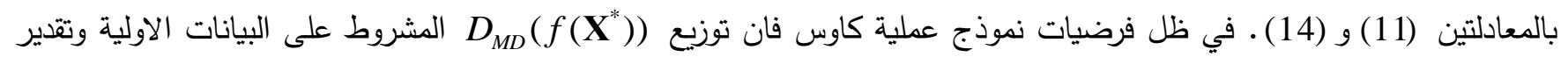

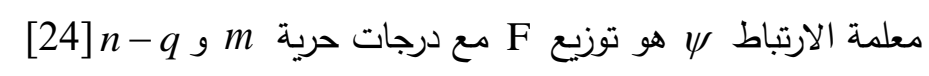

$$
\frac{(n-q)}{m(n-q-2)} D_{M D}\left(f\left(\mathbf{X}^{*}\right)\right) \mid \mathbf{y}, \boldsymbol{\psi} \sim \mathrm{F}_{\mathrm{m}, \mathrm{n}-\mathrm{q}}
$$

تشير القيمة الصغيرة او الكبيرة بشكل غير متوقع لـ (

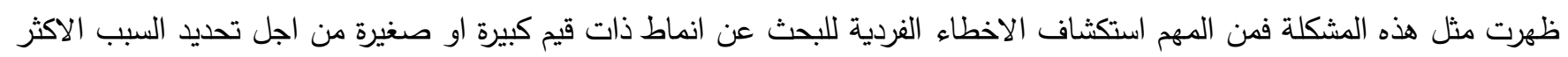
احتمالا للمشكلة وهذا ما سنتطرق اليه القسم التالي. 3.4 تجزئة مصفوفة التباين

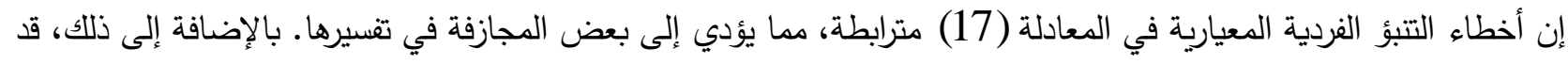

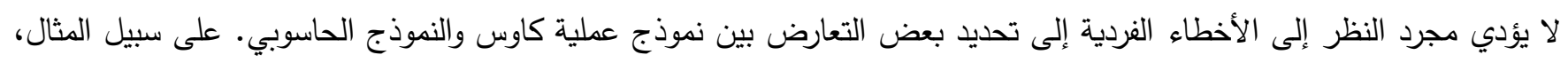

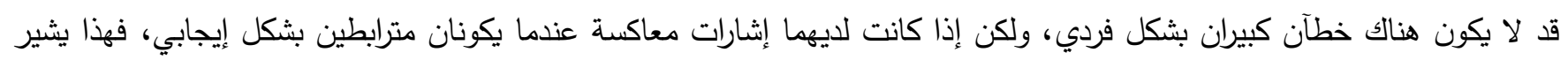

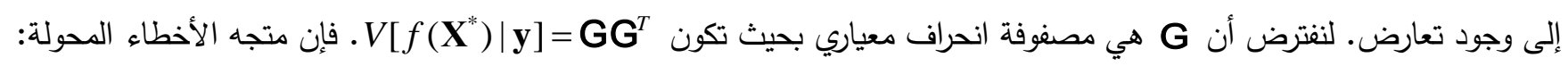

$$
D_{\mathbf{G}}\left(\mathbf{y}^{*}\right)=\mathbf{G}^{-1}\left(\mathbf{y}^{*}-E\left[f\left(\mathbf{X}^{*}\right) \mid \mathbf{y}\right]\right) \text {, }
$$

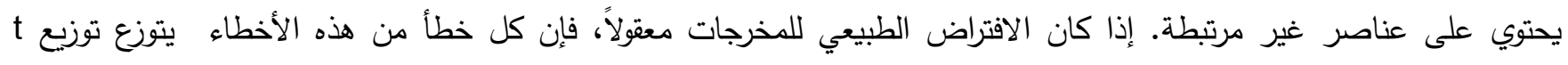

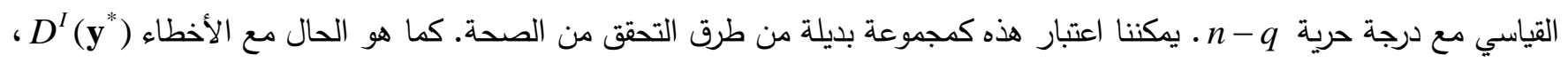

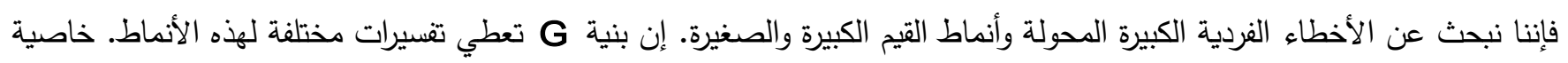

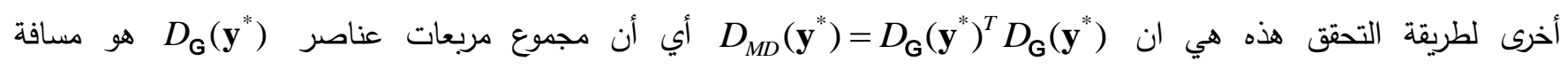

$$
\text { مهالانوبيس. وبالتالي يمكننا تفسير طرق التحقق هذه ان على أنها تجزئة }
$$

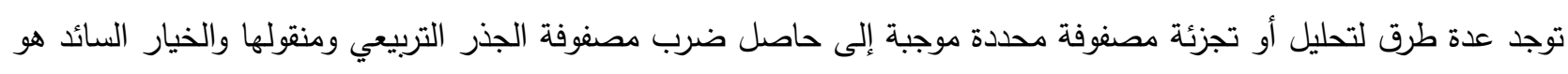

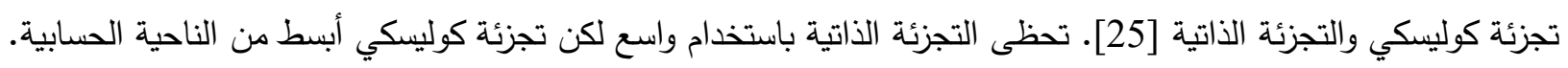

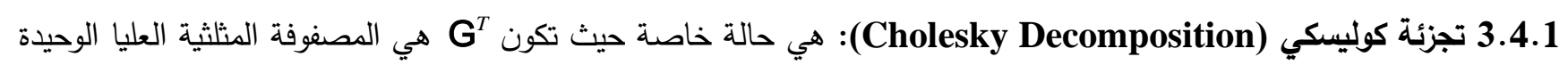

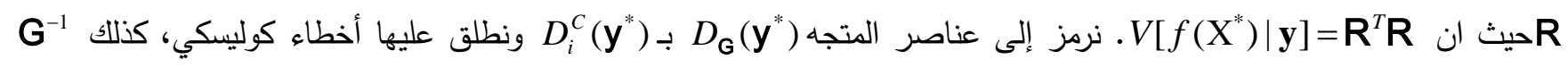

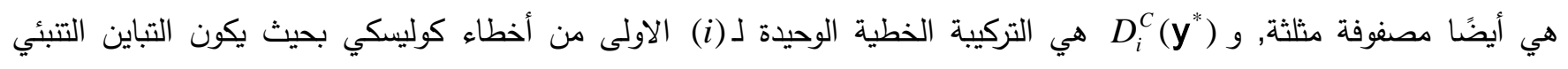

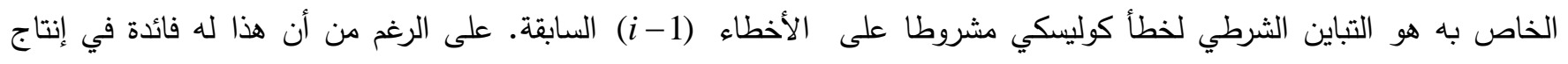

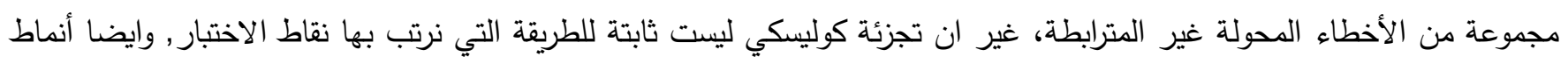
القيم العالية أو المنخفضة ليس لهاء تفسير واضح. 
3.4.2 تجزئة كوليسكي المحوري (Pivoted Cholesky Decomposition)

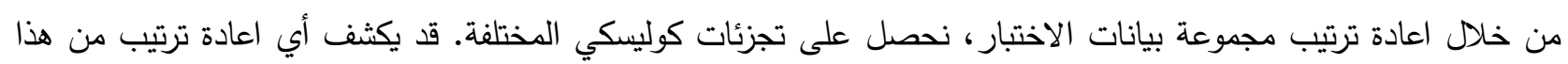
القبيل حالات شاذة مختلفة ولكن للاستفادة من تجزئة (تحلل) كوليسكي، يتم الحصول على طرق التحقق الأكثر فعالية من خلال تبديل

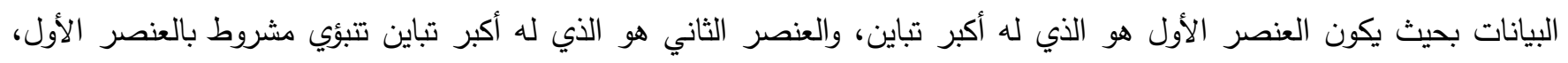
وهكذا. نرمز إلى عناصر المتجه (لإن

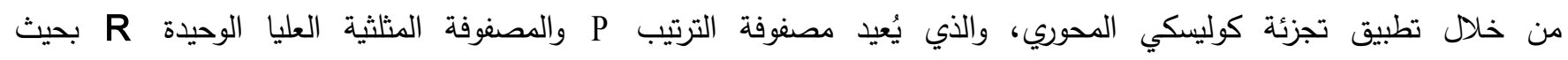

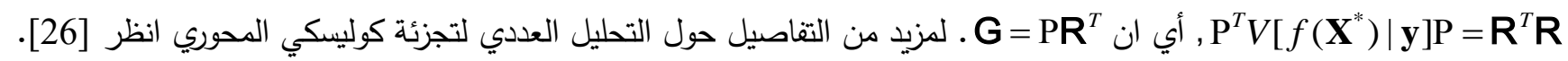

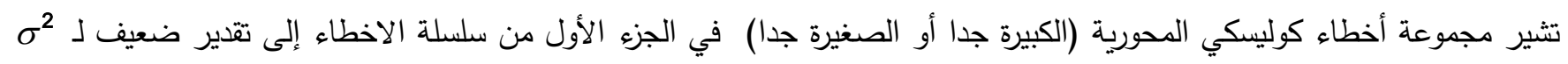

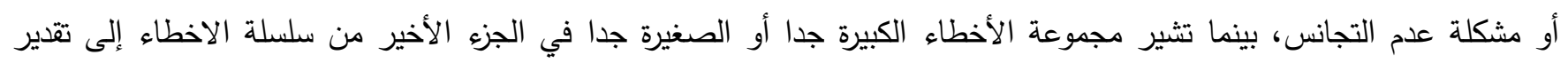
ضعيف لـ ب أو بنية ارتباط غير مناسبة. بالإضافة إلى ذلك، لدينا ميزة أن كل من يجعل من السهل التحقق من الأخطاء الفردية الكبيرة.

3.5

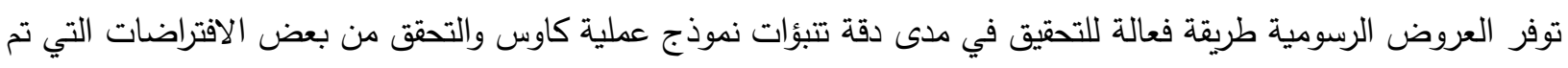

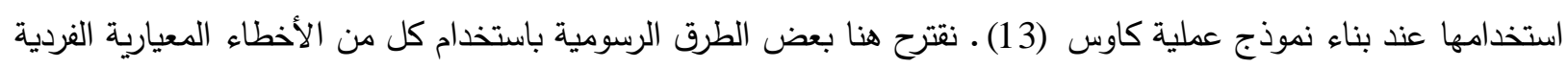

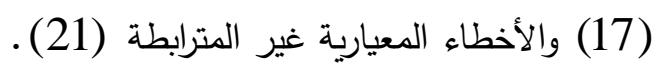

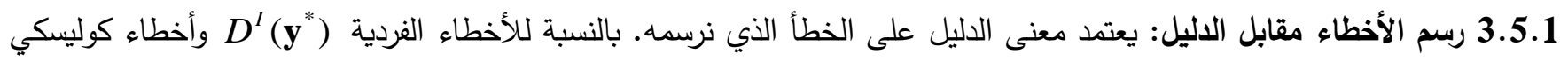
فإن الداليل $D_{i}^{C}\left(\mathbf{y}^{*}\right)$ يعطي ترتيب (

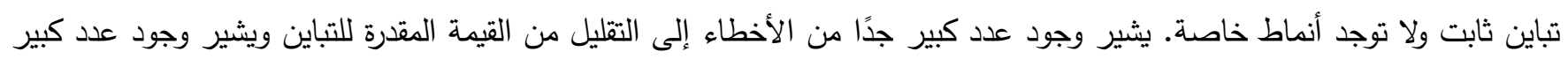

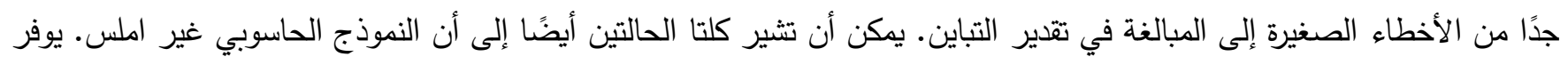

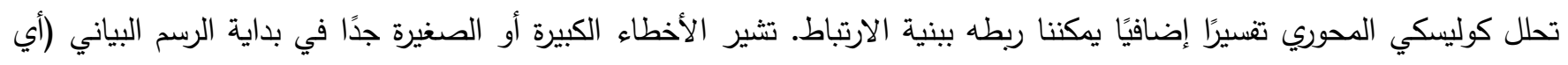

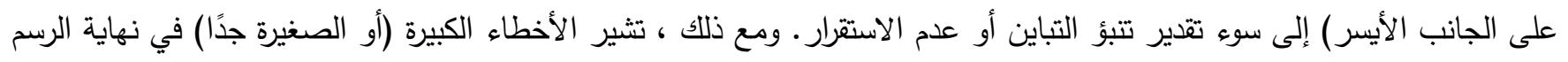

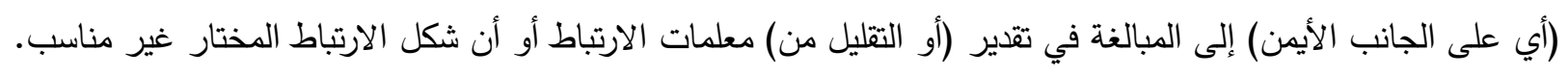

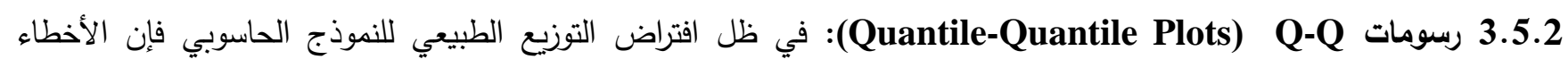

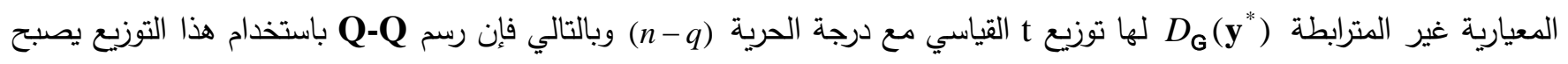

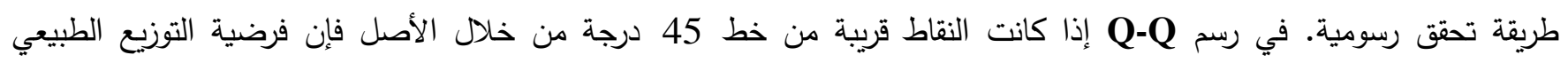

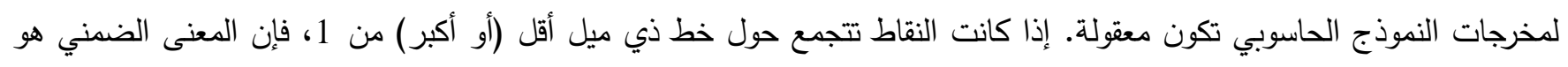

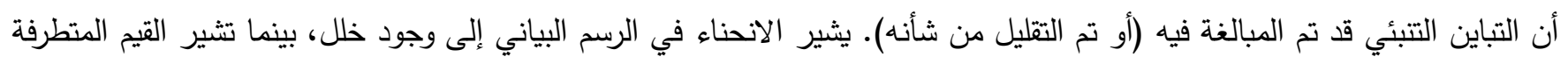

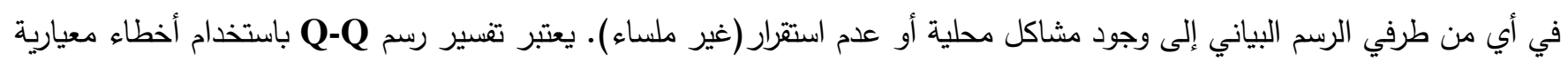

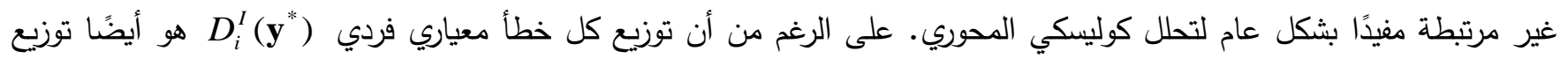

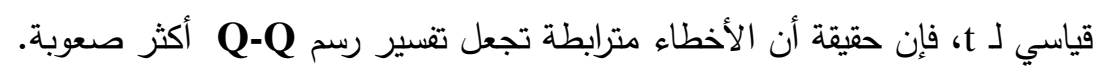


في هذا القسم سيتم اعتبار دالة ذراع الروبوت Robot Arm كمثال على نموذج حاسوبي, دالة ذراع الروبوت Robot Arm استخدمها [27] كمثال على تتفيذ طرق شبه الانحدار واستخدمها [28] كمثال لبناء نموذج كركنك. تعمل دالة ذراع الروبوت على تصميم موضع ذراع الروبوت الذي يحتوي على أربعة أجزاء, يعتبر الكتف ثابتًا عند الأصل في حين أن كل جزء من الأجزاء الأربعة له طول L $L_{i}$

$$
\begin{aligned}
f(\mathbf{x}) & =\left(\omega^{2}+\delta^{2}\right)^{\frac{1}{2}} \\
\omega & =\sum_{i=1}^{4} L_{i} \cos \left(\sum_{j=1}^{i} \vartheta_{j}\right) \quad, \quad \delta=\sum_{i=1}^{4} L_{i} \sin \left(\sum_{j=1}^{i} \vartheta_{j}\right)
\end{aligned}
$$

ناتج دالة ذراع الروبوت هو المسافة بين أصل ونهاية ذراع الروبوت. تحتوي دالة ذراع الروبوت على ثمانية متغيرات إدخال وهي . $\mathbf{x}=\left(\vartheta_{1}, \vartheta_{2}, \vartheta_{3}, \vartheta_{4}, L_{1}, L_{2}, L_{3}, L_{4}\right)$ الأولى والثانية والثالثة والرابعة من الذراع على التوالي, [0,1 والرابعة من الذراع على التوالي. في هذا المثال, تم استخدام مجموعتين من البيانات الاولية لبناء نموذج عملية كاوس لدالة ذراع الروبوت هابـاء تم توليد مجموعة من اربعين نقطة تمثل البيانات الاولية (1) (1) على مخرجات دالة ذراع الروبوت عند هذه النقاط الاربعين, [

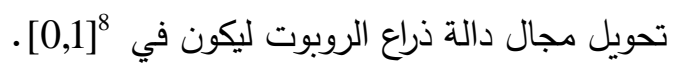
استخدمنا دالة الوسط الخطي

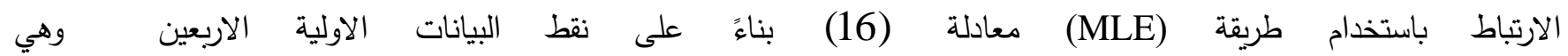

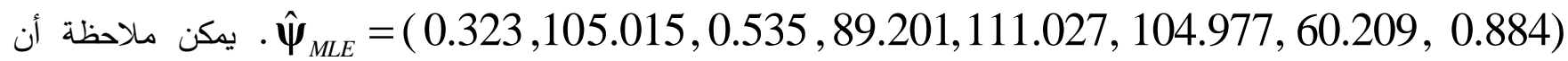
بعض المدخلات لها معاملات ارتباط كبيرة، مما يشير إلى أن دالة النموذج الحاسوبي تكون ملساء أكثر مع هذه المدخلات.

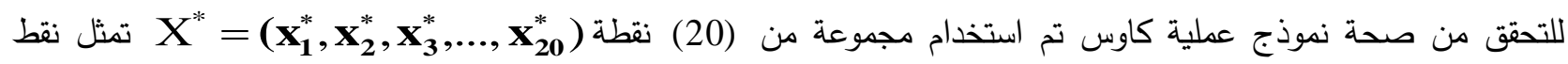
الاختبار والتي تم توليدها ايضا باستخدام (Maximin LHD). بعد ايجاد متجه الوسط التتبئي [ل التتبؤية [ل لنموذج عملية كاوس كما في المعادلتين (11)

\begin{tabular}{|c|c|c|c|c|c|c|c|}
\hline \multicolumn{8}{|c|}{ جدول (1): يوضح مسافة مهالانوبيس المحسوبة وطريقة تحقق مربع كاي لأربعين نقطة من البيانات الاولية ، إلى جانب } \\
\hline & Obs. & Min & 1st Qu & Median & Mean & 3rd Qu & Max \\
\hline$D_{M D}(\bullet)$ & 1120.532 & 5.733 & 14.143 & 18.803 & 20.361 & 24.985 & 81.529 \\
\hline$D_{\chi^{2}}(\bullet)$ & 436.163 & 5.068 & 15.960 & 19.766 & 20.326 & 24.047 & 44.220 \\
\hline
\end{tabular}
لمخرجات الروبوت مقابل التوزيع التتبؤي عند فترة ثقة 0.95.

نلاحظ أن معظم النقاط المتوقعة لا يقع على خط ( y=x) بالاضافة الى ذلك، يبدو أن عدم اليقين(Error Bars) لبعض النقاط كبير نوعا ما مما يشير إلى أن تتبؤات نموذج عملية كاوس هي تقديرات تقريبية غير دقيقة لمخرجات النموذج الحاسوبي بفترة ثقة 
(0.95). باستخدام طرق التحقق من الصحة, تم حساب مسافة مهالانوبيس (Mahalanobis Distance) حسب المعادلة (19)

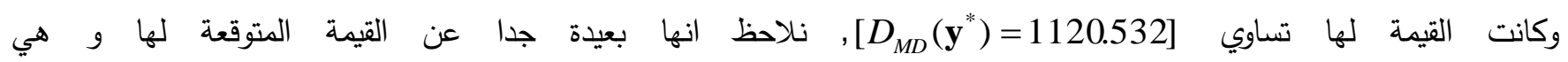
] الحاسوبي ونموذج عمليات كاوس. تم ايضا حساب طريقة تحقق مربع كاي (X)

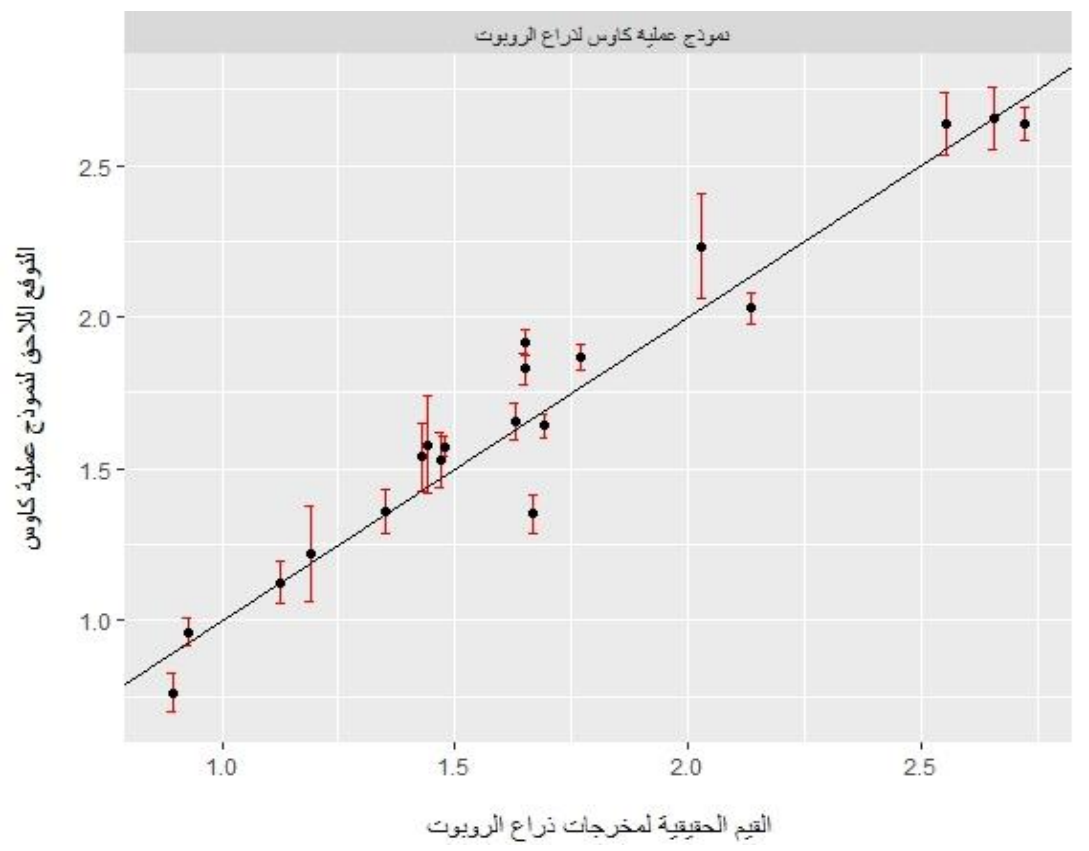

(شكل 1) يمثل القيم الحقيقية لمخرجات الروبوت مقابل التوزيع التنبوي عند فترة ثقة 0.95 بالاعتماد على اربعين نقطة من البيانات الاولية

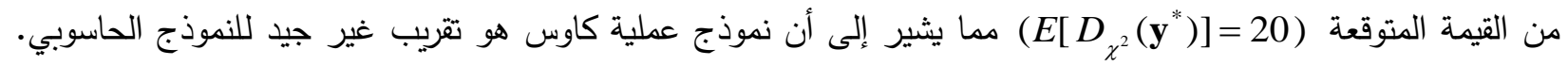
لكن هذه الطريقة للتحقق تجاهل حقيقة أن النواتج مترابطة. استخدمنا طرقاً اخرى للتحقق من الصحة حيث تم حساب الاخطاء المعيارية الفردية بحسب المعادلة (17) كما موضحة

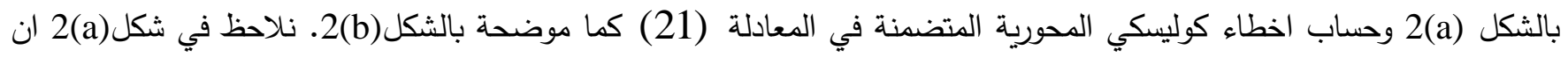
نصف الاخطاء المعيارية تقع في الفترة [2,-2] لكن النصف الاخر منها تقع خارج الفترة وان قسماً من هذه الاخيرة تكون متطرفة مما يشير الى وجود تعارض بين النموذج الحاسوبي و نموذج عملية كاوس, وفي نفس السياق نلاحظ في الشكل (b) (2) ان غالبية اخطاء

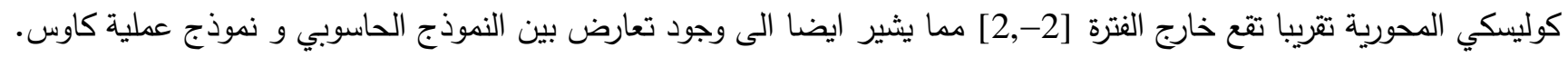

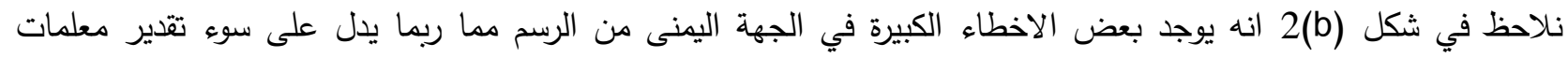

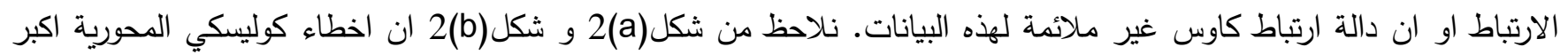
واكثر تطرفا من الاخطاء المعيارية والسبب في ذللك ان اخطاء كوليسكي الححورية مستقلة اما الاخطاء المعيارية فهي مرتبطة. لذلك يكون تفسير اخطاء كوليسكي المحورية اكثر دقة من الاخطاء المعيارية. 

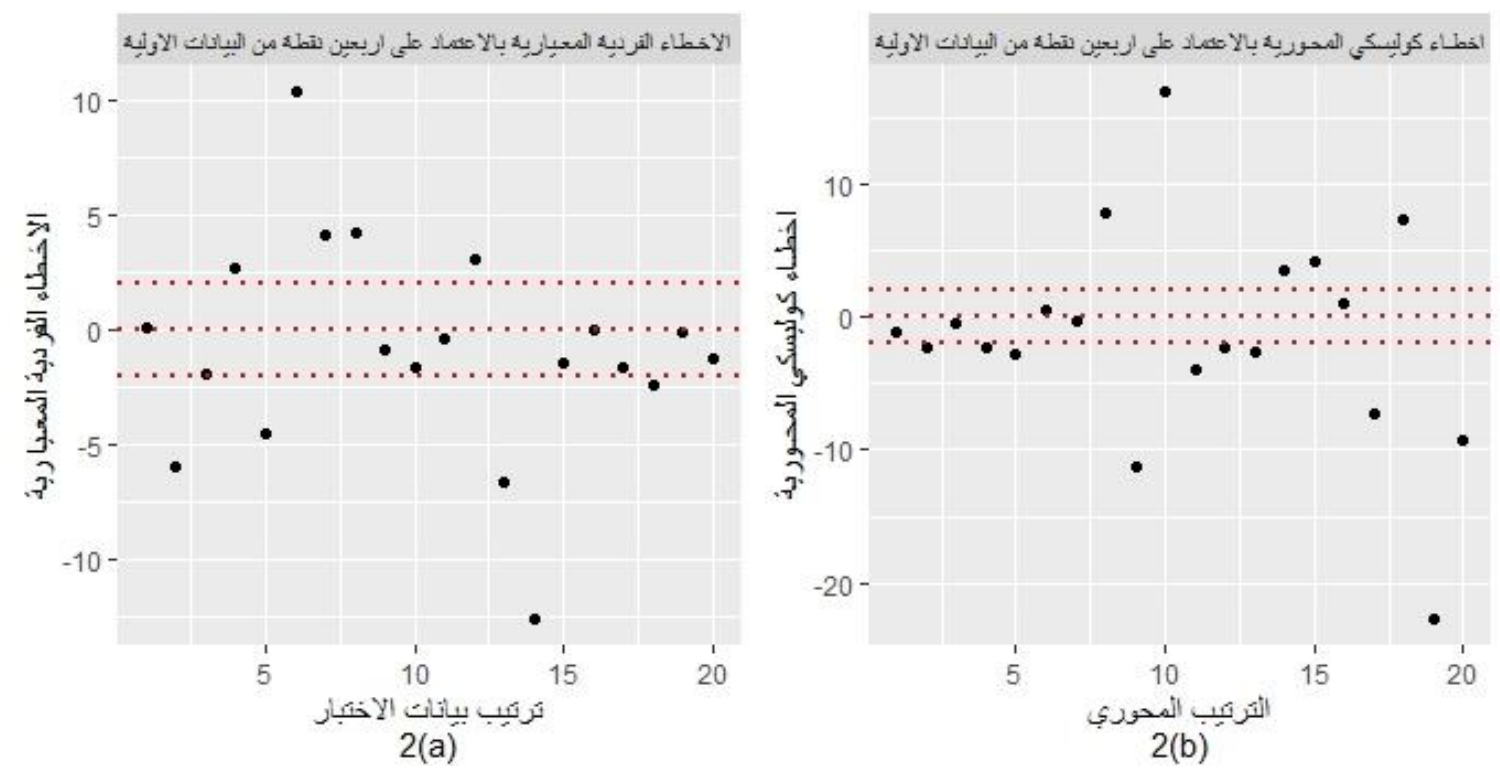

(شكل 2): يوضح شكل (a) الاخطاء المعيارية الفردية مقابل ترتيب بيانات الاختبار

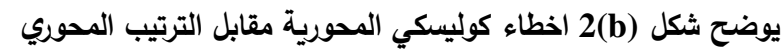

استخدنا رسم Q-Q كطريقة تحقق اضافية من صحة نموذج عملية كاوس كما هو موضح في الثكل(3), حيث يتم عرض أخطاء

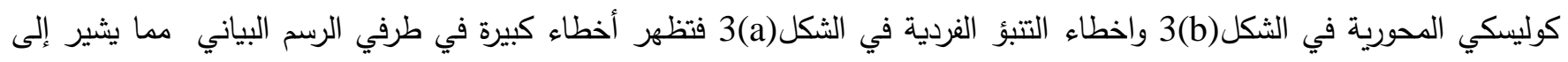

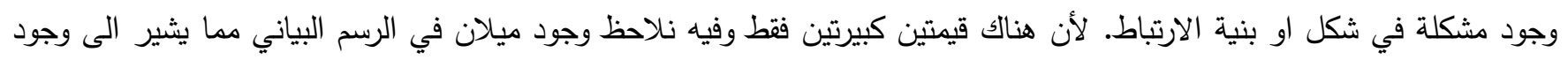

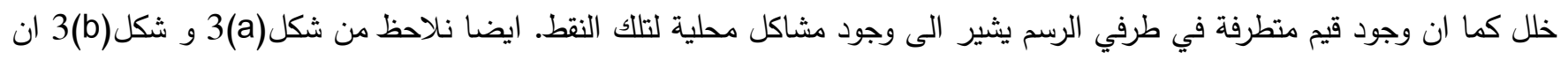

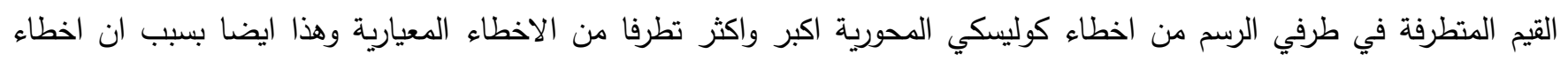
كوليسكي المحورية مستقلة اما الاخطاء المعيارية فهي مرتبطة. كخطوة لاحقة من عملية التحقق تم زيادة الان من عدد البيانات الاولية حيث ولدانا ثمانين البانين نقطة من نقط البيانات الاولية (Maximin LHD) بود ذللك تم الحصول على مخرجات دالة ذراع الروبوت عند هذه النقاط الثمانين, طربن

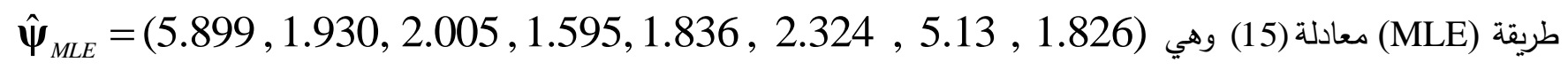

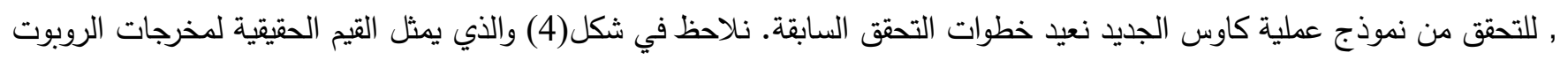

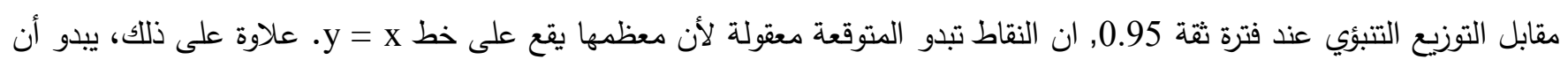

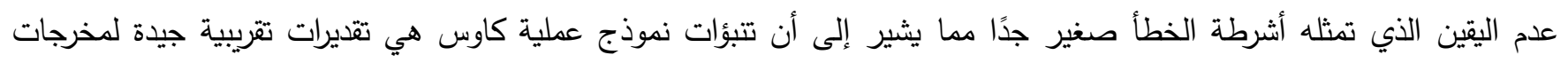

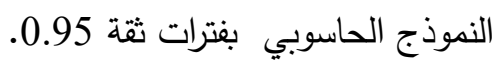


(a)

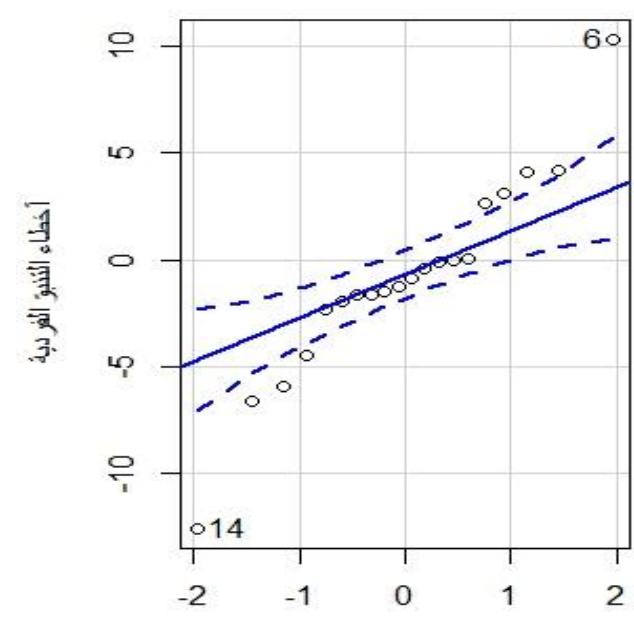

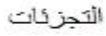

(b)

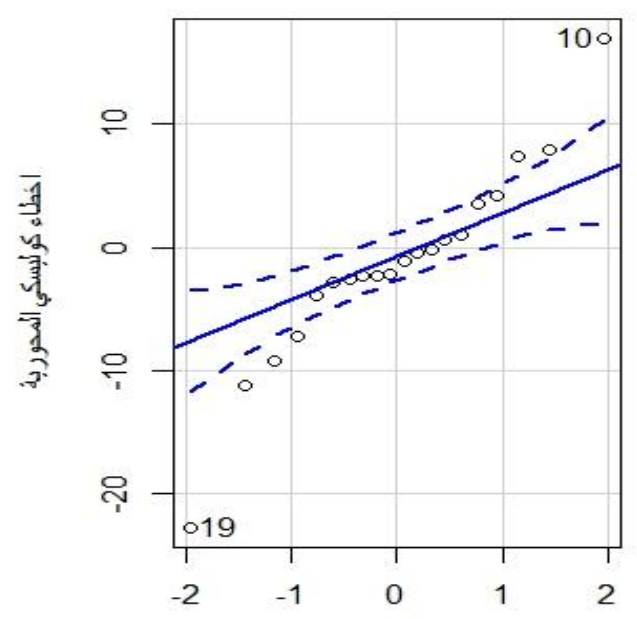

الأنجزئن

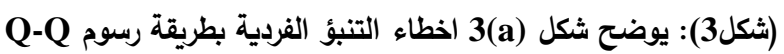

يوضح شكل(b) اخطاء كوليسكي المحورية بطريقة رسوم Q-Q(بالاعتماد على اربعين نقطة من البيانات الاولية)

نلاحظ ايضا بعد حساب مسافة مهالانوبيس (Mahalanobis Distance) وكانت القيمة لها تساوي

[ 23.83374 وجود توافق بين النموذج الحاسوبي ونموذج عمليات كاوس. وجدنا كنلك في طريقة تحقق مريع كاي تكون ذات قيمة اقل قياسا بالمرة السابقة لكنها تكون بعيدة نوعا ما من القيمة المتوقعة (20) طريقة التحقق هذه من صحة نموذج عملية كاوس غير موفقة مع وجود فرضية الترابط بين نواتج النموذج.

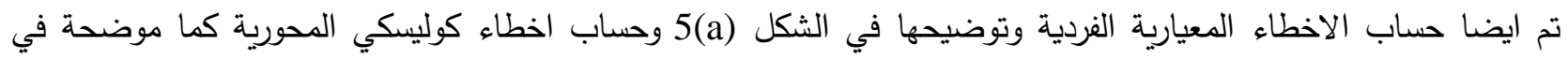

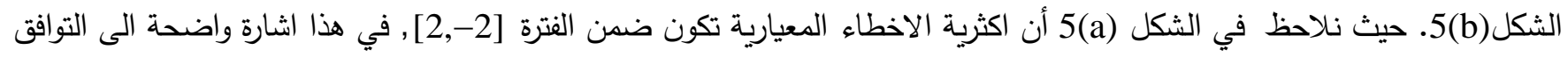

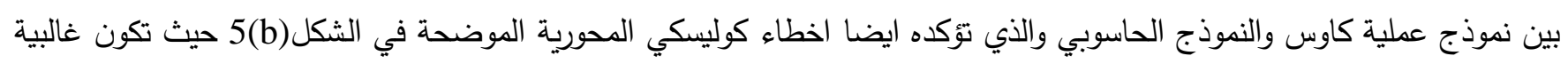
الاخطاء ضمن الفترة [2-,2]. نلاحظ من شكل(a)2 و شكل(b) 2(2) انه يوجد خطآن فقط من اخطاء كوليسكي المحورية يقع خارج الفترة [2,2] بينما يوجد ثلاثة اخطاء معيارية يقع خارج الفترة [2,2] والسبب في ذلك كما ذكرنا سابقا ان اخطاء كوليسكي المحورية مستقلة اما الاخطاء المعيارية فهي مرتبطة.

\begin{tabular}{|c|c|c|c|c|c|c|c|}
\hline \multicolumn{8}{|c|}{ 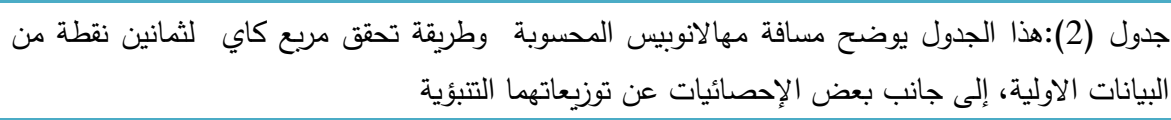 } \\
\hline & & Min & 1st Qu & Median & Mean & $3 \mathrm{rd} \Omega$ & Max \\
\hline & & & & & & & 52.945 \\
\hline$D_{\chi^{2}}(\bullet)$ & 40.97208 & 5.068 & 15.960 & 19.766 & 20.334 & 24.047 & 44.220 \\
\hline
\end{tabular}




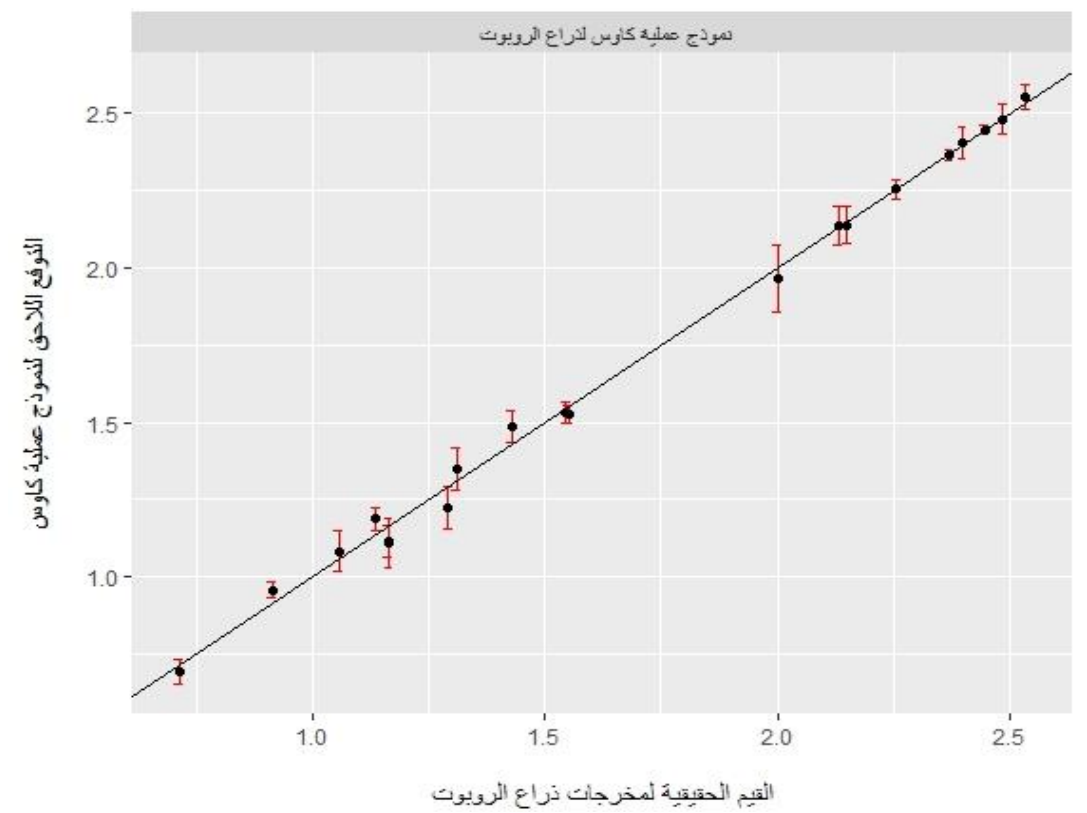

(ثكل 4) يوضح يمثل القيم الحقيقية لمخرجات الروبوت مقابل التوزيع التنبؤي عند فترة ثقة 0.95 بالاعتماد على ثمانين نقطة من البيانات الاولية

لذا فانه من خلال اخطاء كوليسكي المحورية نستطيع تحديد نقاط الاختبار التي قد يكون فيها مشكلة محلية حول تلك ال نقاط. نستخدم رسوم (Q) للتحقق من صحة نموذج عملية كاوس كما هو موضح في (الثكل 6) وفيه نلاحظ ان النقاط تكون قريبة من خط 45 درجة من خلال الأصل وبهذا فإن فرضية التوزيع الطبيعي لمخرجات النموذج الحاسوبي تكون معقول, وبهذا نستطيع القول بانه كلما زاد عدد البيانات الاولية كان الحصول نموذج عملية كاوس متقارب من النموذج الحاسوبي امر ممكن.

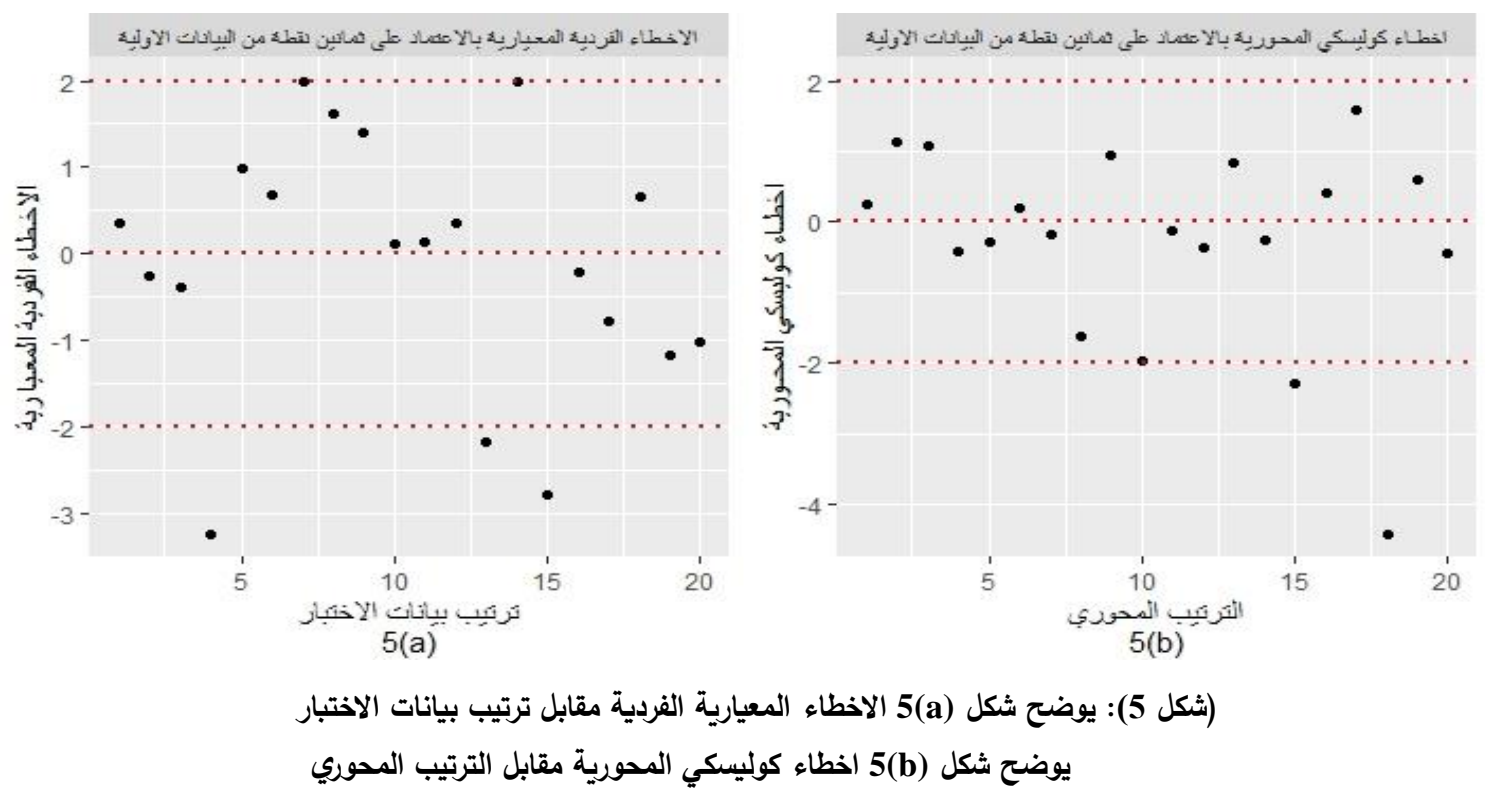


(a)

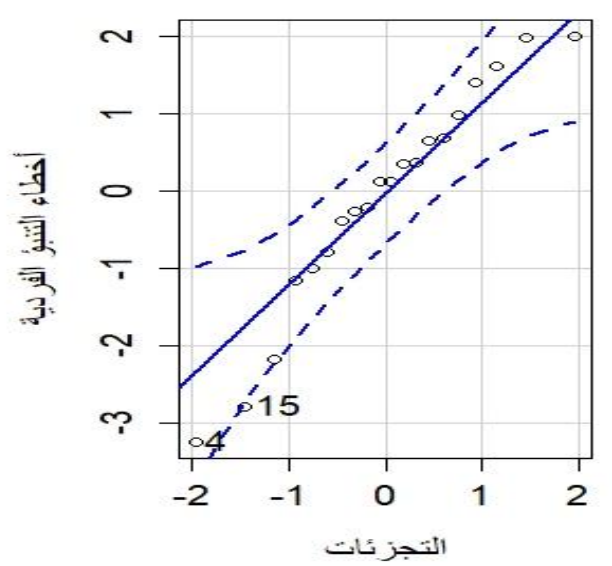

(b)

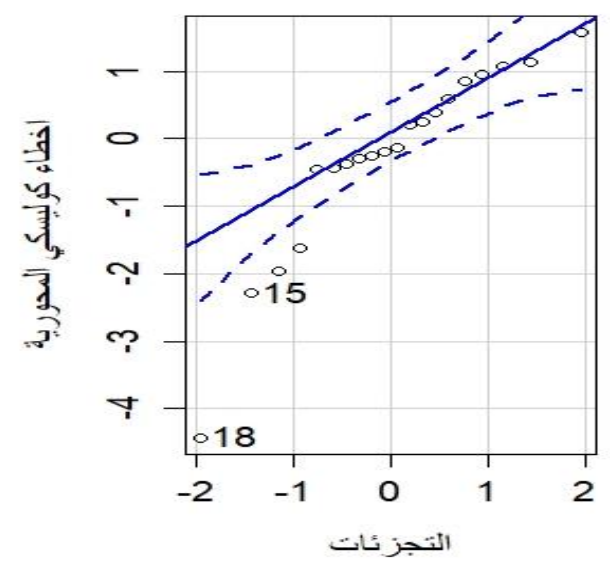

التجزئات

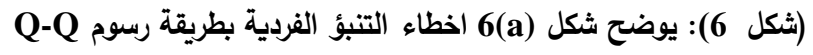

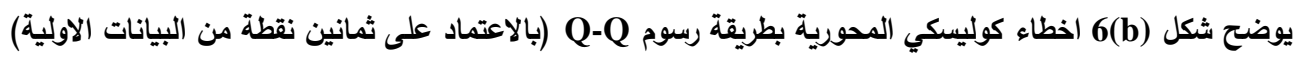

5. - 5 الاستنتاجات

تم في هذا البحث تقديم اسلوب بيز في بناء نماذج عملية كاوس كنماذج بديلة للنماذج الحاسوبية المعقدة, عن طريق توليد بيانات

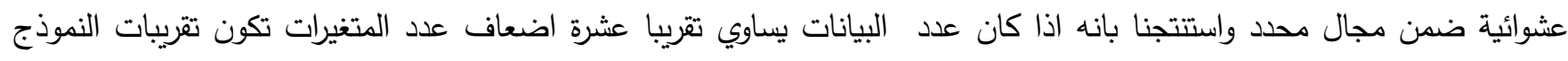

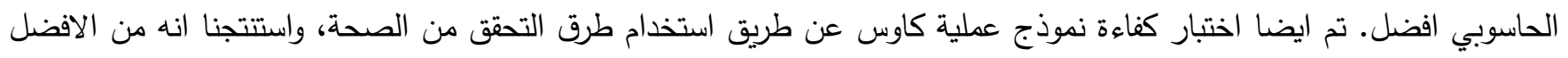

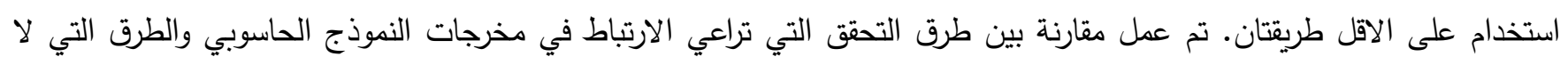
تراعي الارتباط في مخرجات النموذج الحاسوبي. لتوضح نموذج عملية كاوس وطرق التحقق المقترحة، اخذنا مثال حقيقي متمثل بدالة ذراع الروبوت وعملنا على بناء نموذج عملية كاوس خاص به وتم تطبيق طرق التحقق عليه موضحة بالرسوم البيانية. من خلال النتائج

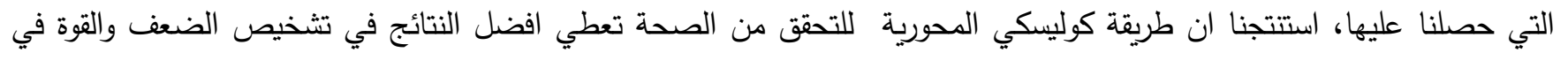
كفاءة نموذج عملية كاوس لنموذج دالة ذراع الروبوت لأنها تولد اخطاء غير مرتبطة مع بعضها. شكر و تقدير (Acknowledgement)

يتقدم الباحثون بالشكر و التقدير الى قسم الرياضيات في كلية التربية للعلوم الصرفة - جامعة الموصل لتقديم التسهيلات اللازمة لاكمال هذا البحث المصادر

[1] J. Sacks, W. J. Welch, T. J. Mitchell, and H. P. Wynn, "Design and analysis of computer experiments," Stat. Sci., pp. 409-423, 1989.

[2] C. Currin, T. Mitchell, M. Morris, and D. Ylvisaker, "A Bayesian approach to the design and analysis of computer experiments”. Technical Report ORNL-6498, Oak Ridge National Laboratory. 1988.

[3] C. Currin, T. Mitchell, M. Morris, and D. Ylvisaker, "Bayesian prediction of deterministic functions, with applications to the design and analysis of computer experiments," J. Am. Stat. Assoc., vol. 86, no. 416, pp. 953-963, 1991.

[4] A. O’Hagan, "Curve fitting and optimal design for prediction," J. R. Stat. Soc. Ser. B, vol. 40, no. 1, pp. 1-24, 1978.

[5] W. J. Welch, R. J. Buck, J. Sacks, H. P. Wynn, T. J. Mitchell, and M. D. Morris, "Screening, predicting, and computer experiments," Technometrics, vol. 34, no. 1, pp. 15-25, 1992. 
[6] J. Oakley and A. O'Hagan, "Bayesian inference for the uncertainty distribution of computer model outputs," Biometrika, vol. 89, no. 4, pp. 769-784, 2002.

[7] A. Saltelli, K. Chan, and E. M. Scott, Sensitivity Analysis. Wiley, 2009.

[8] J. E. Oakley and A. O'Hagan, "Probabilistic sensitivity analysis of complex models: a Bayesian approach,” J. R. Stat. Soc. Ser. B (Statistical Methodol., vol. 66, no. 3, pp. 751-769, 2004.

[9] M. C. Kennedy, C. W. Anderson, S. Conti, and A. O’Hagan, "Case studies in Gaussian process modelling of computer codes," Reliab. Eng. Syst. Saf., vol. 91, no. 10-11, pp. 1301-1309, 2006.

[10] G. S. Kimeldorf and G. Wahba, "A correspondence between Bayesian estimation on stochastic processes and smoothing by splines," Ann. Math. Stat., vol. 41, no. 2, pp. 495-502, 1970.

[11] M. C. Kennedy and A. O'Hagan, "Bayesian calibration of computer models," J. R. Stat. Soc. Ser. B (Statistical Methodol., vol. 63, no. 3, pp. 425-464, 2001.

[12] A. O'Hagan, "Bayesian analysis of computer code outputs: A tutorial," Reliab. Eng. Syst. Saf., vol. 91, no. 10-11, pp. 1290-1300, 2006.

[13] Y. Al-Taweel, "Diagnostics and Simulation-Based Methods for Validating Gaussian Process Emulators" PhD. Thesis, college of science, University of Sheffield, 2018.

[14] M. D. Morris and T. J. Mitchell, "Exploratory designs for computational experiments," J. Stat. Plan. Inference, vol. 43, no. 3, pp. 381-402, 1995.

[15] W. Oberkampf and T. Trucano, "Validation methodology in computational fluid dynamics," in Fluids 2000 Conference and Exhibit, 2000, p. 2549.

[16] M. J. Bayarri et al., "A framework for validation of computer models," Technometrics, vol. 49, no. 2, pp. 138-154, 2007.

[17] J. Rougier, D. M. H. Sexton, J. M. Murphy, and D. Stainforth, “Analyzing the climate sensitivity of the HadSM3 climate model using ensembles from different but related experiments," J. Clim., vol. 22, no. 13, pp. 3540-3557, 2009.

[18] M. Goldstein and J. Rougier, "Bayes linear calibrated prediction for complex systems," J. Am. Stat. Assoc., vol. 101, no. 475, pp. 1132-1143, 2006.

[19] J. Haslett and K. Hayes, "Residuals for the linear model with general covariance structure," J. R. Stat. Soc. Ser. B (Statistical Methodol., vol. 60, no. 1, pp. 201-215, 1998.

[20] R. Fraccaro, R. J. Hyndman, and A. Veevers, "Theory \& Methods: Residual Diagnostic Plots for Checking for Model Mis-specification in Time Series Regression," Aust. N. Z. J. Stat., vol. 42, no. 4, pp. 463-477, 2000.

[21] E. A. Houseman, L. M. Ryan, and B. A. Coull, "Cholesky residuals for assessing normal errors in a linear model with correlated outcomes," J. Am. Stat. Assoc., vol. 99, no. 466, pp. 383-394, 2004.

[22] R. G. Hills and T. G. Trucano, "Statistical validation of engineering and scientific models: Background," Sandia Natl. Lab. Albuquerque, NM, Rep. No. SAND99-1256, 1999.

[23] R. G. Hills and T. G. Trucano, "Statistical Validation of Engineering and Scientific Models: A Maximum Likelihood Based Metric"; TOPICAL, United States 2002.

[24] L. S. Bastos and A. O’Hagan, "Diagnostics for Gaussian process emulators," Technometrics, vol. 51, no. 4, pp. 425-438, 2009.

[25] G. H. Golub and C. F. Van Loan, "Matrix Computations Johns Hopkins University Press," Balt. London, 1996.

[26] N. J. Higham, Accuracy and stability of numerical algorithms. SIAM, 2nd Ed, Philadelphia, 2002.

[27] J. An and A. Owen, "Quasi-regression," J. Complex., vol. 17, no. 4, pp. 588-607, 2001.

[28] Y. H. Al-Taweel and N. Sadeek, "A comparison of different methods for building Bayesian kriging models,” Pakistan J. Stat. Oper. Res., pp. 73-82, 2020. 